\title{
Fluids of rod-like particles near curved surfaces
}

\author{
B. Groh ${ }^{1}$ and S. Dietrich ${ }^{2}$ \\ ${ }^{1}$ FOM Institute for Atomic and Molecular Physics, Kruislaan 407, 1098 SJ Amsterdam, The \\ Netherlands \\ ${ }^{2}$ Fachbereich Physik, Bergische Universität Wuppertal, D-42097 Wuppertal, Germany
} (July 26, 2021)

\begin{abstract}
We study fluids of hard rods in the vicinity of hard spherical and cylindrical surfaces at densities below the isotropic-nematic transition. The Onsager second virial approximation is applied, which is known to yield exact results for the bulk properties in the limit of infinitely thin rods. This approach requires the computation of the one-particle distribution function and of the Mayer function which is greatly facilitated by an appropriate expansion in terms of spherical harmonics. We determine density and orientational profiles as well as the surface tension $\gamma$ as function of the surface curvature radius $R$. Already in the low-density limit of non-interacting rods $\gamma(R)$ turns out to be non-analytic at $1 / R=0$, which prohibits the application of the commonly used Helfrich expansion. The interparticle interaction modifies the behavior of $\gamma(R)$ as compared to the low-density limit quantitatively and qualitatively.
\end{abstract}

PACS numbers: 68.45.-v, 61.30.Gd, 68.10.Cr, 82.70.Dd 


\section{INTRODUCTION}

A fluid of hard rods can be considered as the simplest model for nematic liquid crystals consisting of elongated molecules. In a seminal paper in 1949 Onsager showed [1] that the steric hard-body interactions alone can bring about an isotropic-nematic transition. Although the steric interactions already capture many of the essential features of liquid crystals their actual behavior is complicated by the presence of dispersion forces, flexibility, dipole moments, etc. But for certain colloidal systems of rod-like particles of synthetic or biological origin dissolved in a suitable solvent the hard rod model provides a quantitatively reliable effective description [2]. Among them the ones, which are studied in most detail, are the tabac mosaic virus and the fd-virus with length $(L)$ to diameter $(D)$ ratios $L / D$ of about 17 and 150, respectively [2]. From a theoretical point of view the limit of infinitely thin hard rods is especially interesting because it represents one of the very few cases for which the exact density functional is known [1].

Even more than in simple liquids, which are composed of spherically symmetric particles, surface effects are of great importance for liquid crystals. In the absence of external fields the orientation of the bulk fluid is determined by its interaction with the container walls; this phenomenon is called anchoring [3]. For the simplest case of a hard rod fluid near a planar hard wall, as studied theoretically by Hołyst and Poniewierski [4 6], the wall induces parallel alignment of the nematic director. An isotropic-nematic interface also aligns the nematic phase parallel to the interface for large aspect ratios $L / D$ while a non-trivial tilt angle arises for lower aspect ratios [7]. In view of the substantial technical difficulties which are associated with the theoretical description on a truly microscopic scale, especially for curved surfaces, it is a natural first step to analyze the interface between the isotropic phase and a hard wall. The corresponding density and orientational order profiles near a planar wall have been determined by Poniewierski [8] in the framework of the Onsager theory, who also found indications for spontaneous biaxial order at the surface already below the bulk transition to the nematic phase. Mao et al. have compared this theory with computer simulations for finite aspect ratios $L / D$ [9] and have calculated the depletion force between planar walls or large spheres immersed in a solution of rods [10,11], using the Derjaguin approximation.

In the present work we focus on the orientational and positional order as well as the surface tension near curved hard walls, taking into account the steric interactions between the rods. The curvature has an appreciable effect on the structure and the thermodynamics of the fluid if the radius of curvature $R$ is of the order of the particle length $L$. Accordingly as possible applications one can think of the following systems: (i) rod-like particles confined to the interior of small pores within porous materials; (ii) colloidal suspensions of rods that contain a second, diluted, component of larger, e.g., spherical particles; (iii) membranes, especially vesicles, immersed in colloidal rod solutions, resembling, e.g., solutions of viruses. The curvature dependent surface tension and the depletion forces in case (ii) have been determined by Auvray [12] and Yaman et al. [13, 14] for fluids of non-interacting rods corresponding to the limit of infinite dilution. Their most surprising result was that the surface free energy does not contain a term linear in the curvature $1 / R$ and that the quadratic term has different amplitudes for different signs of the curvature. This non-analyticity prohibits the application of the common Helfrich expansion. Thus for case (iii) above the effect of the 
rods on the elastic properties of the membranes cannot be described by a renormalization of the bending rigidities as it is possible for membranes exposed to a suspension of spherical colloidal particles [13] or polymers [15]. In order to be able to assess the range of validity of the results obtained in the ideal limit of non-interacting rods, in the present paper we tackle the full problem including the inter-particle interactions by employing the Onsager density-functional theory (Sec. III), which yields density profiles (Sec. III) and the surface tension (Sec. $(\nabla)$. Our main results are summarized in Sec. $\nabla$ while technical details are presented in Appendices A and B.

\section{MODEL AND DENSITY-FUNCTIONAL THEORY}

Based on density-functional theory we study a fluid of hard spherocylinders of length $L$ and diameter $D$ in the vicinity of a hard spherical or cylindrical surface of radius $R$ (see Fig. (1). In order to keep the numerical difficulties tractable we restrict ourselves to the limit $D / L \rightarrow 0$ with $R / L$ fixed. The number density of the centers of mass of these thin rods at a point $\mathbf{r}$ with orientation $\omega^{\prime}=\left(\theta^{\prime}, \phi^{\prime}\right)$ is denoted by $\hat{\rho}\left(\mathbf{r}, \omega^{\prime}\right)$. The corresponding grand canonical functional is given by

$$
\begin{aligned}
\beta \Omega\left[\left\{\hat{\rho}\left(\mathbf{r}, \omega^{\prime}\right)\right\}\right]= & \int d^{3} r d \omega^{\prime} \hat{\rho}\left(\mathbf{r}, \omega^{\prime}\right)\left[\ln \left(4 \pi \lambda^{3} \hat{\rho}\left(\mathbf{r}, \omega^{\prime}\right)\right)-1-\beta \mu+\beta V\left(\mathbf{r}, \omega^{\prime}\right)\right] \\
& +\beta F_{e x}\left[\left\{\hat{\rho}\left(\mathbf{r}, \omega^{\prime}\right)\right\}\right] .
\end{aligned}
$$

Here $k_{B} \beta=1 / T$ is the inverse temperature, $\mu$ the chemical potential, $V\left(\mathbf{r}, \omega^{\prime}\right)$ the external potential exerted by the hard wall and $\lambda$ the thermal de Broglie wavelength. Within the Onsager second virial approximation the excess free energy $F_{e x}$ is [1]

$$
\beta F_{e x}\left[\left\{\hat{\rho}\left(\mathbf{r}, \omega^{\prime}\right)\right\}\right]=-\frac{1}{2} \int d^{3} r_{1} d \omega_{1}^{\prime} d^{3} r_{2} d \omega_{2}^{\prime} \hat{\rho}\left(\mathbf{r}_{1}, \omega_{1}^{\prime}\right) \hat{\rho}\left(\mathbf{r}_{2}, \omega_{2}^{\prime}\right) f\left(\mathbf{r}_{12}, \omega_{1}^{\prime}, \omega_{2}^{\prime}\right)
$$

with the interparticle vector $\mathbf{r}_{12}=\mathbf{r}_{2}-\mathbf{r}_{1}$ and the Mayer function $f\left(\mathbf{r}_{12}, \omega_{1}^{\prime}, \omega_{2}^{\prime}\right)$ which for hard particles equals -1 if the two particles overlap and zero otherwise. Onsager demonstrated that this approximation becomes exact in the limit $D / L \rightarrow 0$ for the bulk properties [1], and this is expected to hold also for surface quantities [8,11]. For a planar surface this approach has turned out to be quantitatively reliable for $D / L \lesssim 0.1$ [11].

As mentioned in the introduction, the corresponding problem with non-interacting particles, i.e., in the presence of the hard wall interaction but with $F_{e x}=0$, has been analyzed by Yaman et al. [13,14]. Taking into account the inter-particle interaction increases the complexity considerably due to the ensuing non-locality and the high dimensional integration in Eq. (2)). In the remaining part of this section we evaluate the expression for $F_{e x}$ by exploiting the symmetries of the density profile $\hat{\rho}(\mathbf{r}, \omega)$ using appropriate expansions in terms of spherical harmonics. In Sec. III the thermodynamically stable equilibrium profile is obtained by minimization of the density functional, which amounts to solving an integral equation. The value of the functional at this minimum is the grand canonical potential of the inhomogeneous fluid from which the surface tension at the curved walls is determined in Sec. IV.

The particle orientation is conveniently described in a local reference frame whose polar axis is that wall normal which runs through the rod center and whose $y$ axis, in the case of 
a cylindrical wall, is aligned with the cylinder axis (see Fig. 1). Since we are interested in bulk densities $\rho_{b}$ below the isotropic-nematic transition, i.e., $\rho_{b}<4.2\left(D L^{2}\right)^{-1}$ [1], we may assume that the number density $\rho(r)=\int d \omega^{\prime} \hat{\rho}\left(\mathbf{r}, \omega^{\prime}\right)$ as well as the orientational distribution measured in the local reference system (denoted by $\omega=(\theta, \phi)$ ) depend only on the radial coordinate $r$ in a spherical or cylindrical coordinate system, which allows us to make the following ansatz

$$
\hat{\rho}^{S}(\mathbf{r}, \omega)=\frac{\rho_{b}}{2 \pi} \sum_{l=0}^{\infty} \alpha_{l}(r) P_{l}(\cos \theta)
$$

and

$$
\hat{\rho}^{C}(\mathbf{r}, \omega)=\rho_{b} \sum_{l=0}^{\infty} \sum_{m=-l}^{l} \mu_{l m}(r) Y_{l m}(\omega)
$$

for a sphere $(\mathrm{S})$ and a cylinder $(\mathrm{C})$, respectively. The functions $P_{l}$ and $Y_{l m}$ are Legendre polynomials and spherical harmonics, respectively. For large distances from the wall the fluid is isotropic $\left(\hat{\rho}(\mathbf{r}, \omega)=\rho_{b} / 4 \pi\right)$, so that $\alpha_{l}(r \rightarrow \infty)=\frac{1}{2} \delta_{l, 0}$ and $\mu_{l m}(r \rightarrow \infty)=(4 \pi)^{-1 / 2} \delta_{l m, 00}$. At a sphere the density does not depend on the azimuthal angle $\phi$. At a cylinder the symmetries

$$
\hat{\rho}(r, \theta, \phi)=\hat{\rho}(r, \pi-\theta, \phi)=\hat{\rho}(r, \theta,-\phi)=\hat{\rho}(r, \theta, \pi-\phi)
$$

imply that $\mu_{l m}=0$ if $l$ or $m$ is odd and $\mu_{l m}=\mu_{l m}=\mu_{l m}^{*}$ for $l$ and $m$ even (here and in the following $\bar{m}=-m$ ).

The coordinates $\omega$ can be expressed in terms of the coordinates $\omega^{\prime}$ corresponding to a frame fixed in space by a (position dependent) rotation. Therefore the angular integrations in Eqs. (11) and (2) can be taken also over $\omega$. However, the Mayer function is naturally expressed within a third coordinate system $\hat{\omega}$ given by the interparticle vector $\mathbf{r}_{12}$. In order to perform the angular integrations in the following we will determine the transformation from $\hat{\omega}$ to $\omega$. The definition of the different reference frames is illustrated in Fig. 11.

For any uniaxial molecule the Mayer function can be expanded as [16]

$$
f\left(r_{12}, \hat{\omega}_{1}, \hat{\omega}_{2}\right)=\sum_{l_{1}, l_{2}, m} f_{l_{1} l_{2} m}\left(r_{12}\right) Y_{l_{1} m}\left(\hat{\omega}_{1}\right) Y_{l_{2} \bar{m}}\left(\hat{\omega}_{2}\right)
$$

The solid angles $\hat{\omega}_{i}$ refer to a particle fixed reference system (see Fig. 1) with its $z$ axis parallel to the interparticle vector $\mathbf{r}_{12}$ (and arbitrary $x$ axis). The determination of the expansion coefficients

$$
f_{l_{1} l_{2} m}\left(r_{12}\right)=\int d \hat{\omega}_{1} d \hat{\omega}_{2} f\left(r_{12}, \omega_{12}=0, \hat{\omega}_{1}, \hat{\omega}_{2}\right) Y_{l_{1} m}^{*}\left(\hat{\omega}_{1}\right) Y_{l_{2} \bar{m}}^{*}\left(\hat{\omega}_{2}\right)
$$

is discussed in Appendix A. Spherical harmonics in different reference systems are related via the rotation matrices $D_{n m}^{l}$ [16]:

$$
Y_{l m}\left(\omega_{i}\right)=\sum_{n} D_{n m}^{l}\left(\psi_{i}, \eta_{i}, \chi_{i}\right) Y_{l n}\left(\hat{\omega}_{i}\right), \quad i=1,2
$$


where the Euler angles $\psi_{i}\left(\mathbf{r}_{1}, \mathbf{r}_{2}\right), \eta_{i}\left(\mathbf{r}_{1}, \mathbf{r}_{2}\right)$, and $\chi_{i}\left(\mathbf{r}_{1}, \mathbf{r}_{2}\right)$ describe the rotation of the particle based axes $\left(\hat{\omega}_{i}\right)$ onto the surface normal based axes $\left(\omega_{i}\right)$ (for the definition of the Euler angles see, e.g., Fig. A.6 in Ref. [16]). Inserting Eqs. (3), (6), and (8) into Eq. (2) yields for the spherical case

$$
\begin{aligned}
\beta F_{e x}^{S}= & -\frac{\rho_{b}^{2}}{2 \pi} \sum_{l_{1}, l_{2}, m}\left[\left(2 l_{1}+1\right)\left(2 l_{2}+1\right)\right]^{-1 / 2} \\
& \times \int d^{3} r_{1} d^{3} r_{2} \alpha_{l_{1}}\left(r_{1}\right) \alpha_{l_{2}}\left(r_{2}\right) f_{l_{1} l_{2} m}\left(r_{12}\right) D_{m 0}^{l_{1} *}\left(\psi_{1}, \eta_{1}, \chi_{1}\right) D_{\bar{m} 0}^{l_{2} *}\left(\psi_{2}, \eta_{2}, \chi_{2}\right) .
\end{aligned}
$$

One still has the freedom to fix the orientation of the $y$ axes in the different reference systems. If one chooses them to be all parallel to each other and perpendicular to the plane spanned by $\mathbf{r}_{1}$ and $\mathbf{r}_{2}$ the transformations described by the Euler angles become simple rotations around the $y$ axis so that $\psi_{i}=\chi_{i}=0$. With [16

$$
D_{m 0}^{l *}(0, \eta, 0)=\sqrt{\frac{4 \pi}{2 l+1}} Y_{l m}(\eta, 0)
$$

one finds for a system of radial size $\mathcal{L}$, outside of a spherical cavity of radius $R$

$$
\begin{aligned}
\beta F_{e x}^{S}= & -\left(4 \pi \rho_{b}\right)^{2} \sum_{l_{1}, l_{2}, m}\left[\left(2 l_{1}+1\right)\left(2 l_{2}+1\right)\right]^{-1} \int_{R}^{R+\mathcal{L}} d r_{1} r_{1}^{2} \int_{R}^{R+\mathcal{L}} d r_{2} r_{2}^{2} \alpha_{l_{1}}\left(r_{1}\right) \alpha_{l_{2}}\left(r_{2}\right) \\
& \times \int_{-1}^{1} d \cos \gamma f_{l_{1} l_{2} m}\left(r_{12}\right) Y_{l_{1} m}\left(\eta_{1}, 0\right) Y_{l_{2}} \bar{m}\left(\eta_{2}, 0\right) .
\end{aligned}
$$

The angles $\gamma, \eta_{1}$, and $\eta_{2}$ are those between the vectors $\mathbf{r}_{1}$ and $\mathbf{r}_{2}, \mathbf{r}_{1}$ and $\mathbf{r}_{12}$, and $\mathbf{r}_{2}$ and $\mathbf{r}_{12}$, respectively, and $r_{12}=\left(r_{1}^{2}+r_{2}^{2}-2 r_{1} r_{2} \cos \gamma\right)^{1 / 2}$. If we use $r_{12}$ instead of $\cos \gamma$ as integration variable we finally obtain

$$
\beta F_{e x}^{S}=\frac{1}{2} \rho_{b}^{2} \sum_{l_{1}, l_{2}} \int_{R}^{R+\mathcal{L}} d r_{1} r_{1} \int_{R}^{R+\mathcal{L}} d r_{2} r_{2} \alpha_{l_{1}}\left(r_{1}\right) \alpha_{l_{2}}\left(r_{2}\right) w_{l_{1} l_{2}}\left(r_{1}, r_{2}\right)
$$

with

$$
w_{l_{1}, l_{2}}\left(r_{1}, r_{2}\right)=-\frac{32 \pi^{2}}{\left(2 l_{1}+1\right)\left(2 l_{2}+1\right)} \sum_{m} \int_{\left|r_{1}-r_{2}\right|}^{r_{1}+r_{2}} d r_{12} r_{12} f_{l_{1} l_{2} m}\left(r_{12}\right) Y_{l_{1} m}\left(\theta_{1}, 0\right) Y_{l_{2} \bar{m}}\left(\theta_{2}, 0\right)
$$

and

$$
\cos \eta_{1}=\frac{r_{2}^{2}-r_{1}^{2}-r_{12}^{2}}{2 r_{1} r_{12}}, \quad \cos \eta_{2}=\frac{r_{2}^{2}-r_{1}^{2}+r_{12}^{2}}{2 r_{2} r_{12}}
$$

An equivalent expression for a planar wall with surface area $A$ can be derived along the same lines. In this case the Euler angles are the same for both particles because the direction of the surface normal is the same everywhere. Again one can choose $\psi_{i}=\chi_{i}=0$ and finds

$$
\beta F_{e x}^{P} / A=\frac{1}{2} \rho_{b}^{2} \sum_{l_{1}, l_{2}} \int_{0}^{\mathcal{L}} d z_{1} \int_{0}^{\mathcal{L}} d z_{2} \alpha_{l_{1}}\left(z_{1}\right) \alpha_{l_{2}}\left(z_{2}\right) w_{l_{1} l_{2}}^{P}\left(z_{1}-z_{2}\right) .
$$


where the interaction kernel $w_{l_{1} l_{2}}^{P}$ now depends only on one variable:

$$
w_{l_{1} l_{2}}^{P}\left(z_{12}\right)=-\frac{8 \pi}{\left(2 l_{1}+1\right)\left(2 l_{2}+1\right)} \sum_{m} \int_{\left|z_{12}\right|}^{\infty} d r_{12} r_{12} f_{l_{1} l_{2} m}\left(r_{12}\right) Y_{l_{1} m}(\eta, 0) Y_{l_{2} \bar{m}}(\eta, 0) .
$$

with $\eta=\arccos \left(z_{12} / r_{12}\right)$. It can be shown that $w_{l_{1}, l_{2}}\left(R+z_{1}, R+z_{2}\right)=4 \pi w_{l_{1} l_{2}}^{P}\left(z_{1}-z_{2}\right)+O(1 / R)$ for $z_{1}, z_{2} \ll R$.

The cylindrical case is considerably complicated by the lower symmetry of $\hat{\rho}(r, \omega)$. Using Eqs. (2), (4), and (8) and performing the integrations over $\omega_{1}, \omega_{2}$, as well as $z_{1}$ and $\tilde{\phi}_{1}$, where $\mathbf{r}_{i}=\left(r_{i}, z_{i}, \tilde{\phi}_{i}\right), i=1,2$, in cylindrical coordinates, one obtains

$$
\beta F_{e x}^{C} / H=\frac{1}{2} \rho_{b}^{2} \int_{R}^{R+\mathcal{L}} d r_{1} r_{1} \int_{R}^{R+\mathcal{L}} d r_{2} r_{2} \sum_{l_{1}, l_{2}, m_{1}, m_{2}} \mu_{l_{1} m_{1}}\left(r_{1}\right) \mu_{l_{2} m_{2}}\left(r_{2}\right) w_{l_{1} m_{1} l_{2} m_{2}}\left(r_{1}, r_{2}\right)
$$

with

$$
w_{l_{1} m_{1} l_{2} m_{2}}\left(r_{1}, r_{2}\right)=-2 \pi \sum_{m} \int_{-\infty}^{\infty} d z_{12} \int_{0}^{2 \pi} d \tilde{\phi}_{12} f_{l_{1} l_{2} m}\left(r_{12}\right) D_{m m_{1}}^{l_{1} *}\left(\psi_{1}, \eta_{1}, \chi_{1}\right) D_{\bar{m} m_{2}}^{l_{2} *}\left(\psi_{2}, \eta_{2}, \chi_{2}\right) .
$$

Here $H$ is the macroscopic height of the cylinder and $z_{12}=z_{2}-z_{1}, \tilde{\phi}_{12}=\tilde{\phi}_{2}-\tilde{\phi}_{1}, r_{12}=$ $\left(r_{1}^{2}+r_{2}^{2}+z_{12}^{2}-2 r_{1} r_{2} \cos \tilde{\phi}_{12}\right)^{1 / 2}$. In a rather lengthy calculation the dependence of the Euler angles on $r_{1}, r_{2}, z_{12}$, and $\tilde{\phi}_{12}$ can be worked out by decomposing the rotations that connect the different reference systems into three successive simple rotations around (intermediate) coordinate axes (see App. A.2 in Ref. [16]). It is helpful to use $r_{12}$ and $u=r_{12}^{2}-z_{12}^{2}$ as the integration variables which leads to

$$
\begin{aligned}
w_{l_{1} m_{1} l_{2} m_{2}}\left(r_{1}, r_{2}\right)= & -16 \pi \sum_{m} \int_{\left|r_{1}-r_{2}\right|}^{\infty} d r_{12} r_{12} f_{l_{1} l_{2} m}\left(r_{12}\right) \\
& \times \int_{\left(r_{1}-r_{2}\right)^{2}}^{r_{12}^{2}} d u\left[\left(r_{12}^{2}-u\right)\left(\left(r_{1}+r_{2}\right)^{2}-u\right)\left(u-\left(r_{1}-r_{2}\right)^{2}\right)\right]^{-1 / 2} \\
& \times \cos \left[m\left(\psi_{1}-\psi_{2}\right)+m_{1} \chi_{1}+m_{2} \chi_{2}\right] d_{m m_{1}}^{l_{1}}\left(\eta_{1}\right) d_{\frac{m_{m}}{l_{2}}}\left(\eta_{2}\right) .
\end{aligned}
$$

Here the rotation matrices have been written as [16]

$$
D_{m n}^{l}(\psi, \eta, \chi)=e^{-i m \psi} d_{m n}^{l}(\eta) e^{-i n \chi}
$$

where the functions $d_{m n}^{l}$ can be calculated by using Eq. (A.65) in Ref. [16]. The advantage of Eq. (19) is that the inner integral can be evaluated without the time-consuming calculation of $f_{l_{1} l_{2} m}$. In these variables the Euler angles are

$$
\begin{aligned}
\cos \eta_{1}=\frac{r_{2}^{2}-r_{1}^{2}-u}{2 r_{12} r_{1}} & \cos \eta_{2}=\frac{r_{2}^{2}-r_{1}^{2}+u}{2 r_{12} r_{2}} \\
\tan \psi_{1}=2 r_{1} \Delta \cos \eta_{1} & \tan \psi_{2}=2 r_{2} \Delta \cos \eta_{2} \\
\tan \chi_{1}=-2 r_{1} \Delta & \tan \chi_{2}=-2 r_{2} \Delta
\end{aligned}
$$


with

$$
\Delta=\left(\frac{r_{12}^{2}-u}{\left(\left(r_{1}+r_{2}\right)^{2}-u\right)\left(u-\left(r_{1}-r_{2}\right)^{2}\right)}\right)^{1 / 2} .
$$

For the inside of a sphere (cylinder) the integration range for the radial integrals in Eq. (12) (Eq. (17)) has to be replaced by $[0, R]$.

\section{DENSITY PROFILES}

The equilibrium density profile minimizes the grand-canonical functional, i.e., it is a solution of $\frac{\delta \Omega}{\delta \hat{\rho}(r, \omega)}=0$ under the boundary condition $\hat{\rho}(r, \omega) \rightarrow \rho_{b} / 4 \pi$ for $r \rightarrow \infty$. By using the relation

$$
\frac{\delta \alpha_{l}\left(r^{\prime}\right)}{\delta \hat{\rho}(r, \omega)}=\frac{2 l+1}{2 \rho_{b}} \delta\left(r-r^{\prime}\right) P_{l}(\cos \theta)
$$

and exploiting the symmetry property $w_{l_{1} l_{2}}\left(r_{1}, r_{2}\right)=w_{l_{2} l_{1}}\left(r_{2}, r_{1}\right)$ one finds for the spherical wall the Euler Lagrange equation

$$
4 \pi \lambda^{3} \hat{\rho}(r, \theta)=\exp \left[\beta \mu-\beta V(r, \theta)-\frac{\rho_{b}}{4 \pi r} \sum_{l_{1}, l_{2}} \frac{2 l_{1}+1}{2} P_{l_{1}}(\cos \theta) \int d r^{\prime} r^{\prime} \alpha_{l_{2}}\left(r^{\prime}\right) w_{l_{1} l_{2}}\left(r, r^{\prime}\right)\right] .
$$

With $\hat{\rho}_{0}(r, \theta)=\left(4 \pi \lambda^{3}\right)^{-1} \exp [\beta \mu-V(r, \theta)]$ as the corresponding profile for non-interacting rods at the same chemical potential one finds that

$$
\tilde{\rho}(r, \theta):=\hat{\rho}(r, \theta) / \hat{\rho}_{0}(r, \theta)=: \sum_{l} \beta_{l}(r) P_{l}(\cos \theta)
$$

satisfies

$$
\tilde{\rho}(r, \theta)=\exp \left[-\sum_{l} P_{l}(\cos \theta) p_{l}(r)\right]
$$

with

$$
p_{l}(r)=\frac{\rho_{b}}{4 \pi r} \frac{2 l+1}{2} \sum_{l^{\prime}} \int d r^{\prime} r^{\prime} \alpha_{l^{\prime}}\left(r^{\prime}\right) w_{l l^{\prime}}\left(r, r^{\prime}\right)
$$

(Strictly speaking $\tilde{\rho}$ cannot be defined by Eq. (27) for the forbidden orientations, for which both $\hat{\rho}_{0}$ and $\hat{\rho}$ vanish. Instead we define it by Eqs. (28) and (29) in this region.) The function $\hat{\rho}_{0}(r, \theta)$ equals $\rho_{0} / 4 \pi$ for orientations that are allowed by the hard wall and zero otherwise. The density $\rho_{0}$ corresponding to the chemical potential $\mu$ follows from the bulk limit of the density functional. For an isotropic fluid in a volume $V$ one has

$$
\beta \Omega / V=\beta \omega_{b}=\rho_{b}\left(\ln \lambda^{3} \rho_{b}-1-\beta \mu+\frac{1}{2} \rho_{b}^{2} v_{0}\right)
$$


with

$$
v_{0}=-\frac{1}{(4 \pi)^{2}} \int d^{3} r_{12} d \omega_{1} d \omega_{2} f\left(\mathbf{r}_{12}, \omega_{1}, \omega_{2}\right)=\frac{\pi}{2} D L^{2}
$$

The same equation without the last term holds for the ideal gas limit. Minimization yields $\rho_{0}=\rho_{b} \exp \left(\rho_{b} v_{0}\right)$. The allowed values of $\theta$ for given $r$ and $R$ are determined in Appendix $\mathbb{B}$. Thus based on the known function $\hat{\rho}_{0}(r, x=\cos \theta)$ the coefficients $\alpha_{l}$ in Eq. (29) can be expressed in terms of the coefficients $\beta_{l}$ introduced in Eq. (27):

$$
\alpha_{l}(r)=\frac{2 l+1}{2} \frac{2 \pi}{\rho_{b}} \sum_{l^{\prime}} \beta_{l^{\prime}}(r) \int_{-1}^{1} d x P_{l}(x) P_{l^{\prime}}(x) \hat{\rho}_{0}(r, x)
$$

where the integration over $x$ can be carried out analytically for given $l$ and $l^{\prime}$. This allows one to calculate the coefficients $\beta_{l}$ by solving iteratively the following system of equations together with Eqs. (29) and (32):

$$
\beta_{l}(r)=\frac{2 l+1}{2} \int d x P_{l}(x) \exp \left[-\sum_{l^{\prime}} P_{l^{\prime}}(x) p_{l^{\prime}}(r)\right] .
$$

The advantage of first seeking the solution for $\tilde{\rho}$ instead of $\hat{\rho}$ is that the former function is smoother near the transition from allowed to forbidden orientations and hence can be better approximated with a limited number of Legendre polynomials.

The cylindrical case can be treated completely analogously. With the expansion $\tilde{\rho}(r, \omega)=$ $\sum_{l m} \nu_{l m}(r) Y_{l m}(\omega)$ one obtains

$$
\nu_{l m}(r)=\int d \omega Y_{l m}^{*}(\omega) \exp \left[-\sum_{l^{\prime}, m^{\prime}} Y_{l^{\prime} m^{\prime}}^{*}(\omega) p_{l^{\prime} m^{\prime}}(r)\right]
$$

with

$$
p_{l m}(r)=\frac{\rho_{b}}{2 \pi} \sum_{l^{\prime}, m^{\prime}} \int d r^{\prime} r^{\prime} \mu_{l^{\prime} m^{\prime}}\left(r^{\prime}\right) w_{l m l^{\prime} m^{\prime}}\left(r, r^{\prime}\right)
$$

and

$$
\mu_{l m}(r)=\frac{1}{\rho_{b}} \sum_{l^{\prime}, m^{\prime}} \nu_{l^{\prime} m^{\prime}}(r) \int d \omega Y_{l m}^{*}(\omega) Y_{l^{\prime} m^{\prime}}(\omega) \hat{\rho}_{0}(r, \omega) .
$$

These equations are valid both for the outside and the inside of the sphere or cylinder if the $r^{\prime}$ integrations are taken over the interval $[R, \infty)$ or $[0, R]$, respectively. But we note that the functions $\hat{\rho}_{0}$ have completely different forms in these two cases (see Appendix B). It is assumed that the fluid inside a spherical or cylindrical cavity is in equilibrium with a particle reservoir at the chemical potential $\mu$ corresponding to the bulk density $\rho_{b}$, which is kept fixed when $R$ is varied. For small radii the actual density at the center of the cavity may differ from $\rho_{b}$ although this effect is certainly numerically neglegible in the examined range of radii $|R| \geq 3$. 
In practice we have truncated all $l$ sums at $l_{\max }=10\left(l_{\max }=8\right)$ for spheres (cylinders) and the radial integrals were cut off at a distance $\mathcal{L}=2 L(\mathcal{L}=1.5 L)$ from the wall. Beyond this distance the profile was assumed to take on its bulk value and corresponding asymptotic corrections were added to $p_{l}\left(p_{l m}\right)$ in the vicinity of the cutoff. A step size of $\Delta r=0.02 L(\Delta r=0.03125 L)$ was used for all functions of $r$. First the values of $w_{l_{1} l_{2}}\left(r_{1}, r_{2}\right)$ $\left(w_{l_{1} m_{1} l_{2} m_{2}}\left(r_{1}, r_{2}\right)\right)$ were calculated and stored for all necessary values of $r_{1}$ and $r_{2}$ and of the indices. This step required by far the largest fraction of the computer time. Thereafter for a series of bulk densities $\rho_{b}$ the coefficients $\beta_{l}\left(\nu_{l m}\right)$ were determined by a simple Picard iteration scheme with retardation.

In the following a negative (positive) radius $R$ signifies that the wall curves towards (away from) the fluid, and $z$ is the distance from the surface. As reduced density we employ $\rho^{*}=\rho D L^{2}$; in these units the isotropic-nematic transition takes place at $\rho_{b}^{*} \simeq 4.2$ [1], which provides an upper limit for the present approach because in the nematic phase the orientational structure does not exhibit the symmetries assumed here. A typical density profile $\hat{\rho}(z, \cos \theta)$ outside of a sphere is shown in Fig. 2. For $z<L / 2$ orientations with large $\cos \theta$ are forbidden so that the profile has a discontinuity along the line $\cos \theta=x_{\max }(z)$ determined in Appendix B. When the rods do not interact among each other, i.e., for $\rho_{b} \rightarrow 0$, all allowed orientations have the same probability. The presence of the steric interaction induces a strong increase of the density close to the surface, while there is only a weak dependence on $\cos \theta$ within the allowed region. Orientations near the discontinuity, where one end of the rod touches the wall, are slightly favored. Note that no packing effects are visible. These will occur on the much smaller length scale $D$ and presumably only at much higher densities where the packing fraction $\Phi \sim \rho D^{2} L$ is of order unity. The profiles for other radii, even for the opposite sign of the curvature, look essentially the same. In the latter case there is a very small region close to the surface that is not accessible to any rod center. For a cylindrical wall the profiles also depend on the azimuthal angle, but except very close to surfaces with negative curvatures this dependence is very weak and a plot of $\hat{\rho}^{C}(z, \cos \theta, \phi)$ for any fixed $\phi$ looks very similar to Fig. 2 .

The normalized orientationally averaged number density is defined by

$$
n(z)=\int d \omega \hat{\rho}(z, \omega) / \rho_{b}
$$

This function increases for small $z$ up to $z=L / 2$ where it exhibits a cusp and then rapidly decreases to its bulk limit 1 which is essentially reached already at $z=L$. As shown in Fig. 3 within the examined range of curvatures $(|R| / L \gtrsim 3)$ it depends only slightly on $R$. If $1 / R$ is decreased $n(z)$ becomes smaller for $z / L \lesssim 0.27$ and larger for $z / L \gtrsim 0.27$. The results for the cylinder lie between those for the planar wall $(R=\infty)$ and for a sphere with the same radius. Due to the finite step size $\Delta r$ and the steepness of $\hat{\rho}(z, \omega)$ the raw data for $n(z)$ exhibit visible kinks at $z=n \Delta r$ for small integers $n$. These have been removed from Fig. 3 by fitting of an appropriate smooth function to the data.

We define position dependent orientational order parameters as

$$
Q_{l m}(z)=\frac{1}{\rho_{b} n(z)} \int d \omega Y_{l m}^{*}(\omega) \hat{\rho}(z, \omega) .
$$

For the sphere due to the azimuthal symmetry one has $Q_{l m}=0$ for $m \neq 0$. The lowest nontrivial order parameter $Q_{20}(z)$ is plotted in Figs. G and 5. As $\hat{\rho}(z, \omega) /\left(\rho_{b} n(z)\right) \simeq 1$ for $z \rightarrow 0$ 
and in this limit only $\theta=\pi / 2$ is allowed it follows that $Q_{20}(z \rightarrow 0)=-\sqrt{5 / 8 \pi}=-0.3154$. Negative values of $Q_{20}$ indicate that the rods are preferentially aligned parallel to the surface which is of course enforced by the wall. The inter-particle interactions tend to align the rods also for $z>L / 2$, where they cannot directly touch the wall, and increase the alignment for $z<L / 2$ (see Fig. (1). The alignment is stronger for positive than for negative curvature (see Fig. 5).

The biaxiality of the orientational distribution at a cylinder is measured by $Q_{22}(z)$. Positive (negative) values correspond to a preferential orientation perpendicular (parallel) to the cylinder axis. If the orientational distribution is sharply peaked at $\theta=\pi / 2$ and $\phi=0$ (or $\phi=\pi / 2) Q_{22}$ takes on its maximum (minimum) value $Q_{22}= \pm \sqrt{15 / 32 \pi}=$ \pm 0.3863 . The results for cylinders of radius $R / L= \pm 5$ are displayed in Fig. 6. As expected, particles inside a cylinder orient themselves mainly parallel to the cylinder axis, the more the stronger the interactions are. With increasing bulk density the decay of $Q_{22}$ towards the bulk value 0 becomes significantly slower, which probably signals the onset of the formation of a nematic wetting layer upon approaching the isotropic-nematic transition. This interpetration is supported by the fact that the iterations did not converge for $\rho_{b}^{*} \gtrsim 3.5$. For this density range spontaneous biaxial orientational order has also been predicted at a planar surface 88. For positive curvature $Q_{22}$ has the opposite sign and a much lower absolute value. The limit $z \rightarrow 0$ for $Q_{22}$ cannot be determined rigorously in this case because the allowed region in $\omega$ space does not reduce to a single point so that $Q_{22}(z \rightarrow 0)$ still depends on an unknown function of $\phi$. Only in the ideal case $\rho_{b} \rightarrow 0$ this function is constant so that $Q_{22}(z \rightarrow 0)=\sqrt{5 / 96 \pi}=0.1288$. Here, the effect of the interactions is to increase the probability for orientations parallel to the axis so that $Q_{22}$ may even become negative.

\section{SURFACE TENSION}

The curvature dependent surface tension $\gamma(R)$ is that contribution to the grand canonical potential which scales with the surface area of the confining wall. In order to determine this quantity for positive curvature one has to consider systems of finite size $\mathcal{L}$ in the radial direction. However, such systems necessarily contain a second, isotropic liquid - vacuum interface generated by the cutoff yielding the corresponding artificial surface contribution $\gamma_{v a c}$. Hence for a sphere we have

$$
4 \pi R^{2} \gamma^{S}(R)=\lim _{\mathcal{L} \rightarrow \infty}\left[\Omega(R, \mathcal{L})-\frac{4 \pi}{3}\left((R+\mathcal{L})^{3}-R^{3}\right) \omega_{b}-4 \pi(R+\mathcal{L})^{2} \gamma_{\text {vac }}^{S}(R+\mathcal{L})\right]
$$

where $\omega_{b}=-p$ (see Eq. (30)) is the bulk grand canonical potential density and $p$ the bulk pressure.

The vacuum surface tension can be obtained separately by considering the one-surface problem of a sphere of radius $R+\mathcal{L}$ filled completely with an isotropic fluid and in contact with the vacuum. In this case the grand canonical potential is the sum of a bulk term and a surface term proportional to $\gamma_{v a c}$ which leads to

$$
4 \pi(R+\mathcal{L})^{2} \beta \gamma_{\text {vac }}^{S}(R+\mathcal{L})=-\frac{1}{8} \rho_{b}^{2} \int_{0}^{R+\mathcal{L}} d r r \int_{R+\mathcal{L}}^{\infty} d r^{\prime} r^{\prime} w_{00}\left(r, r^{\prime}\right)
$$


In the deriving this expression we have used the relation

$$
\frac{1}{4 \pi r} \int_{0}^{\infty} d r^{\prime} r^{\prime} w_{00}\left(r, r^{\prime}\right)=4 v_{0}
$$

which can be proven using Eqs. (13) and (31). After some algebra one finally obtains

$$
\begin{aligned}
4 \pi R^{2} \beta \gamma^{S}(R)= & 4 \pi \int_{R}^{\infty} d r r^{2}\left\{2 \pi \int_{-1}^{1} d x \hat{\rho}(r, x)\left[\ln 4 \pi \hat{\rho}(r, x) \lambda^{3}-1-\beta \mu\right]-\rho_{b}\left(\ln \rho_{b} \lambda^{3}-1-\beta \mu\right)\right\} \\
& +\frac{1}{2} \rho_{b}^{2} \int_{R}^{\infty} d r_{1} r_{1}\left[-4 \pi r_{1} v_{0}+\int_{R}^{\infty} d r_{2} r_{2} \sum_{l_{1}, l_{2}} \alpha_{l_{1}}\left(r_{1}\right) \alpha_{l_{2}}\left(r_{2}\right) w_{l_{1} l_{2}}\left(r_{1}, r_{2}\right)\right]
\end{aligned}
$$

Inside a sphere the fluid volume is finite which does not allow to carry out the thermodynamic limit. Instead we define $\gamma^{S}$ as

$$
4 \pi R^{2} \gamma^{S}(R)=\Omega(R)-\frac{4 \pi}{3}|R|^{3} \omega_{b}
$$

The resulting expression for $\gamma(R)$ is identical to Eq. (42), but with all radial integrations spanning the interval from 0 to $|R|$ instead of from $R$ to $\infty$. If one uses the fact that the equilibrium profile solves the Euler-Lagrange equation Eq. (26) and that the bulk density satisfies the equation $\ln \rho_{b} \lambda^{3}=\beta \mu-\rho_{b} v_{0}$ these results can be cast into the following simpler form:

$$
\begin{aligned}
4 \pi R^{2} \beta \gamma^{S}(R)= & 4 \pi \int d r r^{2}\left[1-2 \alpha_{0}(r)\right] \\
& -\frac{1}{2} \rho_{b}^{2} \int d r_{1} r_{1}\left[-4 \pi r_{1} v_{0}+\int d r_{2} r_{2} \sum_{l_{1}, l_{2}} \alpha_{l_{1}}\left(r_{1}\right) \alpha_{l_{2}}\left(r_{2}\right) w_{l_{1} l_{2}}\left(r_{1}, r_{2}\right)\right]
\end{aligned}
$$

with different integration limits for the outside and inside, as stated above. However, one should keep in mind that Eq. (42) represents the surface contribution to the density functional and is minimized by the equilibrium profile whereas Eq. (44) only applies to the equilibrium solution. In practice the calculation of the surface tension via both formulas provides a helpful check of the numerical procedure.

The corresponding expression for the cylinder is

$$
2 \pi R \gamma^{C}(R)=\lim _{\mathcal{L} \rightarrow \infty}\left[\Omega(R, \mathcal{L}) / H-\pi\left((R+\mathcal{L})^{2}-R^{2}\right) \omega_{b}-2 \pi(R+\mathcal{L}) \gamma_{\text {vac }}^{C}(R+\mathcal{L})\right] .
$$

No truncation in the axial direction is needed because $\Omega$ is evidently proportional to the macroscopic height $H$. Here we could confirm the analogue of Eq. (41), i.e.,

$$
\int_{0}^{\infty} d r^{\prime} r^{\prime} w_{0000}\left(r, r^{\prime}\right)=8 \pi^{2} v_{0}
$$


only numerically. The resulting expressions for the surface tension, which with the modifications quoted above are also valid for the inside of a cylinder, are

$$
\begin{aligned}
2 \pi R \beta \gamma^{C}= & 2 \pi \int_{R}^{\infty} d r r\left\{\int d \omega \hat{\rho}(r, \omega)[\ln 4 \pi \hat{\rho}(r, \omega)-1-\beta \mu]-\rho_{b}\left(\ln \rho_{b} \lambda^{3}-1-\beta \mu\right)\right\} \\
& +\frac{1}{2} \rho_{b}^{2} \int_{R}^{\infty} d r_{1} r_{1}\left[-2 \pi v_{0}+\int_{R}^{\infty} d r_{2} r_{2} \sum_{l_{1}, l_{2}, m_{1}, m_{2}} \mu_{l_{1} m_{1}}\left(r_{1}\right) \mu_{l_{2} m_{2}}\left(r_{2}\right) w_{l_{1} m_{1} l_{2} m_{2}}\left(r_{1}, r_{2}\right)\right]
\end{aligned}
$$

and, at equilibrium,

$$
\begin{aligned}
2 \pi R \beta \gamma^{C}= & 2 \pi \int_{R}^{\infty} d r r \rho_{b}\left[1-\sqrt{4 \pi} \mu_{00}(r)\right] \\
& -\frac{1}{2} \rho_{b}^{2} \int_{R}^{\infty} d r_{1} r_{1}\left[-2 \pi v_{0}+\int_{R}^{\infty} d r_{2} r_{2} \sum_{l_{1}, l_{2}, m_{1}, m_{2}} \mu_{l_{1} m_{1}}\left(r_{1}\right) \mu_{l_{2} m_{2}}\left(r_{2}\right) w_{l_{1} m_{1} l_{2} m_{2}}\left(r_{1}, r_{2}\right)\right] .
\end{aligned}
$$

We remark that the surface tension depends on the assumed position of the actual surface [17], i.e., on the definition of what is denoted as the volume of the sphere or cylinder, which is not uniquely determined. A different choice for this position would alter the volumes and surface areas occuring in Eq. (39) and thereby in general lead to a different value of $\gamma$. For the planar case this change is $\Delta \gamma=p \Delta x$, where $\Delta x$ is the shift in the surface position, while more complicated relations emerge for curved surfaces, which may significantly change the curvature dependence of what is denoted as the surface tension. On the other hand, experimentally observable quantities do not depend on this arbitrariness of assigning a particular value to the volume of the cavity. For the thin rods we employed the natural definition that the defining surface is given by the position of the rod ends at closest approach. But already for hard spheres, or for rods of finite thickness, there are at least two possible "natural" definitions (see, e.g., Figs. 1 and 14 in Ref. 18]).

The surface tension $\gamma_{0}$ in the ideal gas limit is obtained from Eqs. (44) and (48) by neglecting the interaction contributions and by inserting the ideal profiles $\hat{\rho}_{0}(r, \omega)$ from Appendix B. This results in

$$
\frac{\beta \gamma_{0}^{S}}{\rho_{0}}=\frac{1}{R^{2}} \int d r r^{2}\left(1-2 \alpha_{0}(r)\right)=\frac{1}{R^{2}} \int d r r^{2}\left(1-x_{\max }(r)\right)
$$

for a sphere (with integration limits for $R \lessgtr 0$ as described above) and

$$
\frac{\beta \gamma_{0}^{C}}{\rho_{0}}= \begin{cases}\frac{1}{R} \int_{R}^{\infty} d r r\left[1-\frac{2}{\pi} \int_{0}^{1} d x \phi_{c}(r, x)\right], & R>0 \\ \frac{1}{|R|} \int_{0}^{|R|} d r r\left[1-\int_{0}^{1} d x\left(1-\frac{2}{\pi} \phi_{c}(r, x)\right)\right], & R<0\end{cases}
$$

for a cylinder. This limit has been discussed extensively by Yaman et al. [13, 14], who found the surprising result $\beta \gamma_{0} / \rho_{0}=L / 4$ for rods outside any convex body, while this expression is modified inside a sphere or a cylinder, so that 


$$
\frac{\beta \gamma_{0}^{S}}{\rho_{0}}= \begin{cases}\frac{L}{4}-\frac{L^{3}}{48 R^{2}}, & R<0 \\ \frac{L}{4}, & R>0\end{cases}
$$

and

$$
\frac{\beta \gamma_{0}^{C}}{\rho_{0}}=\left\{\begin{array}{ll}
\frac{L}{4}-\frac{L^{3}}{128 R^{2}}+O\left(R^{-3}\right), & R<0 \\
\frac{L}{4}, & R>0
\end{array} .\right.
$$

We have confirmed these results (analytically for the sphere, numerically for the cylinder) by using Eqs. (49) and (50). The most interesting aspect of these findings is that the surface tension is not analytical at $1 / R=0$ which may lead to unexpected behavior of membranes immersed in colloidal rod suspensions.

We have determined the surface tension in the presence of the interparticle interactions for a series of bulk densities and radii. The results are shown in Fig. 7 as function of $\rho_{b}$ for fixed $R$ and in Fig. 8 as function of $R$ for fixed $\rho_{b}$. For each data point four (spheres) or three (cylinders) numerical calculations with different values of the cutoff $l_{\max }$ were performed. The results were extrapolated to $l_{\max }=\infty$ using a fit function linear or quadratic in $1 / l_{\max }$. The differences betweeen the values at the largest $l_{\max }$ and the extrapolation become considerable $\left[\Delta(\beta \gamma) /\left(\rho_{b} L\right) \simeq 0.02\right]$ at large densities. From comparison of the results obtained by quadratic and linear extrapolation we estimate the error of $\beta \gamma /\left(\rho_{b} L\right)$ to be 0.01 for $\rho_{b}^{*}=4$ but only 0.001 for $\rho_{b}^{*} \leq 2$. Finally we have interpolated smoothly between the 13 data points taken for each radius. In the case of a cylinder at the highest density $\rho_{b}^{*}=3$ the result obtained separately for the special case of a planar wall lies slightly (by 0.003 in the units used here) above the almost linear curve through the other points. A possible explanation for this observation is that at the planar wall a uniaxial orientational distribution has been assumed while the actual equilibrium profile might exhibit a small spontaneous biaxiality as found at the cylindrical walls. Therefore the planar wall result has not been used for the interpolation scheme in this case.

Figure 7 shows the surface tension divided by the density to allow for a better comparison with the ideal rods results that predict a density independent constant for this ratio. In all cases the interaction significantly increases this quantity, by up to $50 \%$ for the intermediate density $\rho_{b}^{*}=2$. In the upper density range saturation or the formation of a maximum are observed. The results for a planar wall are in good agreement with those obtained by Mao et al. [11], who effectively used the same theory but a different numerical method. For almost all densities the surface tension is higher for negative than for positive curvature, in contrast to the behavior at $\rho_{b} \rightarrow 0$. In the latter case $\gamma$ is constant for $R>0$; the slight $R$ dependence for $R<0$ is hardly visible on the scale of Fig. 8. On the other hand, for densities of the order of 1 in reduced units the dependence on $R$ is dominated by a term linear in $1 / R$ that is absent in the ideal limit. The dependence on $R$ becomes stronger and less linear with increasing density, especially for the spherical case.

Due to the limited number of radii for which calculations were performed we are not able to decide whether the small discontinuity of the second derivative of $\gamma(1 / R)$ at $1 / R=0$ that occurs in the ideal limit persists also at finite bulk densities. However, our data do not preclude this possibility. It is commonly assumed that the surface free energy density for a general surface with principal curvatures $1 / R_{1}$ and $1 / R_{2}$ has the Helfrich form [19]

$$
\gamma\left(1 / R_{1}, 1 / R_{2}\right)=\gamma^{P}+2 \kappa\left(\frac{1}{2}\left(\frac{1}{R_{1}}+\frac{1}{R_{2}}\right)-c_{0}\right)^{2}+\bar{\kappa} \frac{1}{R_{1} R_{2}}+\cdots
$$


with the stiffness coefficients $\kappa$ and $\bar{\kappa}$ and the spontaneous curvature $c_{0}$. Inter alia, this form predicts that the contribution to $\gamma(1 / R)$ linear in $1 / R$ for a sphere is twice that for a cylinder. From our numerical results we found that this relation is approximately fulfilled at low densities, but there are substantial deviations from it at higher densities. Moreover, the Helfrich expression cannot be applicable for all signs of the curvatures already in the ideal limit due to the aforementioned non-analyticity of $\gamma^{S}(1 / R)$ and $\gamma^{C}(1 / R)$.

We conclude that for phenomena for which the curvature dependence of the surface tension matters the non-interacting approximation is qualitatively wrong already at relatively low densities (compared to the transition density to the nematic phase) and at any fixed curvature it produces rather large quantitative errors in the absolute value of $\gamma$.

\section{SUMMARY}

For an isotropic fluid of needle-like hard spherocylinders of length $L$ near hard spherical or cylindrical walls we have obtained the following main results:

1. Due to the interparticle interactions the probability of finding a particle with a given orientation is strongly increased close to the wall when compared to the bulk fluid. At a given distance $z$ of the center of mass from the wall orientations for which one end of the rod touches the wall are most favorable (see Fig. 21).

2. Since the range of accessible orientations decreases when the particle approaches the wall the orientationally averaged density vanishes for $z \rightarrow 0$. It exhibits a cusp at $z=L / 2$ where the rods lose contact with the surface (Fig. 3).

3. The parallel alignment favored by the surface decays more slowly when the bulk density is increased (Fig. 1) and is stronger if the wall curves towards the fluid instead of away from it (Fig. 5).

4. A cylindrical wall curving towards the fluid induces biaxial orientational order with preferential alignment parallel to the cylinder axis. With increasing bulk density a nematic wetting layer develops in this case (see Fig. 6).

5. The density and curvature dependences of the wall-fluid surface tension are shown in Figs. 7 and 8. In contrast to the results for non-interacting particles given by Eqs. (51) and $(\overline{52})$ the surface tension decreases with increasing curvature and exhibits a linear behavior around the planar limit which leads to a spontaneous curvature of a membrane away from a fluid of rod-like (colloidal) particles.

\section{APPENDIX A: DETERMINATION OF THE EXPANSION COEFFICIENTS OF THE MAYER FUNCTION}

Equation (7) serves as the starting point which defines the expansion coefficients $f_{l_{1} l_{2} m}(r)$. As far as the azimuthal angles are concerned the integrand depends only on $\hat{\phi}_{12}=\hat{\phi}_{2}-\hat{\phi}_{1}$ because 


$$
Y_{l m}(\theta, \phi)=k_{l m} P_{l m}(\cos \theta) e^{-i m \phi},
$$

where the coefficients $k_{l m}$ relating the spherical harmonics to the associated Legendre functions $P_{l m}$ are given by

$$
k_{l m}=(-1)^{m}\left(\frac{2 l+1}{4 \pi} \frac{(l-m) !}{(l+m) !}\right)^{1 / 2} \text { for } m \geq 0, \quad k_{l \bar{m}}=(-1)^{m} k_{l m} .
$$

Therefore after the substitutions $\hat{\phi}_{s}=\frac{1}{2}\left(\hat{\phi}_{1}+\hat{\phi}_{2}\right)$ and $\hat{\phi}_{12}=\hat{\phi}_{2}-\hat{\phi}_{1}$ the integral over $\hat{\phi}_{s}$ renders a factor $2 \pi$. In Eq. (7) we now perform the integrations in the following order:

$$
\begin{aligned}
f_{l_{1} l_{2} m}(r)= & 2 \pi k_{l_{1} m} k_{l_{2}} \bar{m} \int_{-1}^{1} d \cos \hat{\theta}_{1} \int_{-1}^{1} d \cos \hat{\theta}_{2} \int_{0}^{2 \pi} d \hat{\phi}_{12} f\left(r, \hat{\theta}_{1}, \hat{\theta}_{2}, \hat{\phi}_{12}\right) P_{l_{1} m}\left(\cos \hat{\theta}_{1}\right) \\
& \times P_{l_{2} m}\left(\cos \hat{\theta}_{2}\right) e^{i m \hat{\phi}_{12}} .
\end{aligned}
$$

In the limit $D / L \rightarrow 0$ the two rods overlap only in a small range $\Delta \hat{\phi}_{12}$ around $\hat{\phi}_{12}=0$ or $\hat{\phi}_{12}=\pi$, approximately given by $\Delta \phi_{12}=2 D / p$ where $p$ is the distance between the intersection point and the line joining the centers of the rods, as shown in Fig. 9. From this figure one easily derives $p=r /\left(\cot \theta_{1}-\cot \theta_{2}\right)$; based on appropiately modified figures one finds that this expression is also valid if $\theta_{1}>\pi / 2$ or $\theta_{2}<\pi / 2$. Therefore it is sufficient to replace the $\hat{\phi}_{12}$ integration by the factor $\Delta \hat{\phi}_{12}$, to replace the integrand by its value at $\hat{\phi}_{12}=0$ or $\pi$, and to examine only the overlap of two infinitely thin rods in the $\hat{x} \hat{z}$ plane. In integrations over the full spatial angles $\hat{\omega}_{i}$ we assign an intrinsic directionality to the rods such that their "front ends" point into the direction $\left(\hat{\theta}_{i}, \hat{\phi}_{i}\right)$ and their "rear end" into the direction $\left(\pi-\hat{\theta}_{i}, 2 \pi-\hat{\phi}_{i}\right)$. In order to calculate $f_{l_{1} l_{2} m}$ it is sufficient to integrate over the configurations with overlap of the two front ends, where necessarily $\hat{\phi}_{12} \simeq 0$. One can easily show by appropriate substitutions that the other three possibilities for overlap yield exactly the same contribution to the total integral, so that one finds

$$
\begin{aligned}
f_{l_{1} l_{2} m}(r)= & -16 \pi k_{l_{1} m} k_{l_{2}} \frac{D}{r} \int_{-1}^{1} d x_{1} P_{l_{1} m}\left(x_{1}\right) \\
& \times \int_{x_{\min }\left(r / L, x_{1}\right)}^{x_{\max }\left(r / L, x_{1}\right)} d x_{2} P_{l_{2} m}\left(x_{2}\right)\left(\frac{x_{1}}{\sqrt{1-x_{1}^{2}}}-\frac{x_{2}}{\sqrt{1-x_{2}^{2}}}\right) .
\end{aligned}
$$

Here $x_{\min }$ and $x_{\max }$ denote the smallest and largest value of $x_{2}=\cos \hat{\theta}_{2}$ for given $x_{1}=\cos \hat{\theta}_{1}$ and $r / L$ for which the front halves of the rods overlap, as shown for an example in the inset of Fig. 10. They can be determined by tedious but straightforward geometry, which yields two basic formulas for the $x$ value at touching, depending on whether the end of rod 1 touches rod 2 or vice versa:

$$
x_{t}^{(1)}\left(r^{\prime}, x\right)=\frac{2 r^{\prime}-x}{\sqrt{1-2 r^{\prime} x+r^{\prime 2}}} \quad x_{t}^{(2, \pm)}\left(r^{\prime}, x\right)=2 r^{\prime}\left(1-x^{2}\right) \pm x \sqrt{1-4 r^{\prime 2}\left(1-x^{2}\right)}
$$

with $r^{\prime}=r / L$. As illustrated in Fig. 10 the following regions in the $\left(r^{\prime}, x\right)$ plane must be distinguished: 

$\mathrm{A}$, for $0 \leq r^{\prime} \leq 1 / 2$ and $r^{\prime} \leq x \leq 1: x_{\min }=x_{t}^{(1)}$ and $x_{\max }=1$
$\mathrm{B}$, for $1 / 2 \leq r^{\prime} \leq 1$ and $r^{\prime} \leq x \leq 1: x_{\min }=x_{t}^{(1)}$ and $x_{\max }=x_{t}^{(2,+)}$,
$\mathrm{C}$, for $1 / 2 \leq r^{\prime} \leq 1 / \sqrt{2}$ and $r \sqrt{1-1 / 4 r^{\prime 2}} \leq x \leq r^{\prime}: x_{\min }=x_{t}^{(2,-)}$ and $x_{\max }=x_{t}^{(2,+)}$,
$\mathrm{D}$, for $0 \leq r^{\prime} \leq 1 / 2$ and $-1 \leq x \leq r^{\prime}: x_{\min }=x_{t}^{(2,-)}$ and $x_{\max }=1$.

For given $l$ and $m$ the integration over $x_{2}$ in Eq. (A4) can be carried out analytically. In the remaining numerical integration special care must be taken for $r^{\prime}<1 / 2$ due to the square root singularity at $x_{1}=1$. All coefficients $f_{l_{1} l_{2} m}$ are of the order of $D / L$ and vanish for $r / L>1$. They diverge for $r / L \rightarrow 0$, but they appear only in the product $r f_{l_{1} l_{2} m}(r)$ (see Eqs. (13), (16), and (19)) which is finite in this limit. For two finite values of $D / L$ the coefficients $f_{l_{1} l_{2} m}$ have been calculated by Moore and McMullen [7].

A useful check of the numerical results is obtained from the observation that the excluded volume $v_{e x}(\cos \gamma)$ for fixed angle $\gamma$ between the particle axes is related to the Mayer function via $v_{e x}(\cos \gamma)=-\int d^{3} r_{12} f\left(\mathbf{r}_{12}, \omega_{1}, \omega_{2}\right)$ from which one derives

$$
\int_{-1}^{1} d \cos \gamma P_{l}(\cos \gamma) v_{e x}(\cos \gamma)=\frac{2}{2 l+1} \sum_{m}(-1)^{m+1} \int_{0}^{\infty} d r r^{2} f_{l l m}(r) .
$$

The left hand side can easily be determined using the well-known result (see, e.g., Ref. [2]) $v_{e x}(\cos \gamma)=2 D L^{2}|\sin \gamma|+O\left(D^{2} L\right)$ and thus provides a sum rule for the second moments of the expansion coefficients. Our numerical results passed this check.

\section{APPENDIX B: DENSITY PROFILES FOR NON-INTERACTING RODS}

In the ideal limit $\hat{\rho}(r, \omega)$ adopts the constant value $\rho_{0} / 4 \pi$ for orientations $\omega$ that are allowed by the presence of the hard wall and vanishes otherwise. Thus it is sufficient to determine the limiting orientations for which the rod just touches the wall. At a planar surface the maximum allowed value for $x=\cos \theta$ is $x_{\max }=2 z / L$ for $z \leq L / 2$ while the rod cannot touch the wall for $z>L / 2$ so that $x_{\max }(z \geq L / 2)=1$. At all surfaces the minimum value of $x$ clearly is $-x_{\max }$ because of the head-tail symmetry. Thus in the following it is sufficient to consider positive $x$.

\section{Outside a sphere}

As illustrated in Fig. 11(a), there are two different ways how a rod can touch the outside of a sphere: when the rod is sufficiently far from the surface its end touches the wall upon rotation, whereas when it is close to the wall at contact it will touch it tangentially. The crossover between these two regions takes place at $r_{c}=\sqrt{R^{2}+L^{2} / 4}$ and straightforward geometrical reasoning yields

$$
x_{\text {max }}=\left\{\begin{array}{cl}
1, & r \geq R+L / 2 \\
\left(L^{2} / 4-R^{2}+r^{2}\right) /(r L), & r_{c} \leq r \leq R+L / 2 \\
\sqrt{r^{2}-R^{2}} / r, & R \leq r \leq r_{c}
\end{array}\right.
$$




\section{Inside a sphere}

In this case there is a minimum distance $r_{c}=\sqrt{R^{2}-L^{2} / 4}$ from the surface beyond which all orientations are forbidden. In the accessible region $|R|-L / 2 \leq r \leq r_{c}$ one finds [see Fig. 11(a)]

$$
x_{\max }=\frac{R^{2}-L^{2} / 4-r^{2}}{r L} .
$$

\section{Outside a cylinder}

For a cylinder the profile depends in addition on the azimuthal angle $\phi$ which we always measure from the axis that is perpendicular to both the cylinder axis and the surface normal. Thus here we have to determine the range $I_{\phi}$ of allowed values of $\phi$ for fixed $r$ and $\cos \theta$. If the rod touches the cylinder at the angle $\phi_{c} \in[0, \pi / 2]$ then due to symmetry one has $I_{\phi}=\left[0, \phi_{c}\right] \cup\left[\pi-\phi_{c}, \pi+\phi_{c}\right] \cup\left[2 \pi-\phi_{c}, 2 \pi\right]$. With the help of Fig. 11(b) one finds for contact between the rod end and the surface

$$
\cos \phi_{c}=\frac{2 \sqrt{R^{2}-(r-L / 2 \cos \theta)^{2}}}{L \sin \theta}
$$

whereas for tangential contact one has

$$
\cos \phi_{c}=\cot \theta \frac{R^{2}}{\sqrt{r^{2}-R^{2}}} .
$$

By determining the transitions between these two cases as well as those to the ranges $I_{\phi}=\emptyset$ and $I_{\phi}=[0,2 \pi]$ we are led to distinguish the following cases (see Fig. 12):

$\mathrm{A}, R \leq r \leq R+L / 2$ and $0 \leq \cos \theta \leq 2(r-R) / L: \cos \phi_{c}=0$,

$\mathrm{B}, r_{c}=\sqrt{R^{2}+L^{2} / 4} \leq r \leq R+L / 2$ and $2(r-R) / L \leq \cos \theta \leq\left(L^{2} / 4-R^{2}+r^{2}\right) /(L r)$ : $\cos \phi_{c}$ as given by Eq. (B3),

$\mathrm{C}, R \leq r \leq r_{c}$ and $2(r-R) / L \leq \cos \theta \leq 2\left(r^{2}-R^{2}\right) /(L r): \cos \phi_{c}$ as given by Eq. (B3),

$\mathrm{D}, R \leq r \leq r_{c}$ and $2\left(r^{2}-R^{2}\right) /(L r) \leq \cos \theta \leq \sqrt{r^{2}-R^{2}} / r: \cos \phi_{c}$ as given by Eq. (B 4 ).

In the remaining region within $R \leq r \leq R+L / 2$ all $\phi$ values are forbidden, while obviously for $r \geq R+L / 2$ all orientations are allowed.

\section{Inside a cylinder}

In contrast to the previous case, here the accessible $\phi$ range is centered around the cylinder axis, i.e., it has the form $I_{\phi}=\left[\phi_{c}, \pi-\phi_{c}\right] \cup\left[\pi+\phi_{c}, 2 \pi-\phi_{c}\right]$. Since tangential contact is not possible, the classification is a little bit simpler. In the region of interest $|R|-L / 2 \leq r \leq|R|$ we obtain: 
$\mathrm{A}$, for $\cos \theta \geq 2(r-|R|) / L: \cos \phi_{c}=\pi / 2$,

$\mathrm{B}$, for $r \geq r_{c}$ and $\cos \theta \leq 2(r-|R|) / L$ or for $r \leq r_{c}$ and $\left(R^{2}-L^{2} / 4-r^{2}\right) /(L r) \leq \cos \theta \leq$ $2(r-|R|) / L:$

$$
\cos \phi_{c}=\frac{2 \sqrt{R^{2}-(r+L / 2 \cos \theta)^{2}}}{L \sin \theta}
$$

$\mathrm{C}$, for $r \leq r_{c}$ and $\cos \theta \leq\left(R^{2}-L^{2} / 4-r^{2}\right) /(L r): \cos \phi_{c}=0$.

In Fig. 13 we compare the accessible orientational space in these four cases for the same radius $|R| / L=3$ and the same distance from the surface $z / L=0.2$. Naturally this space is largest outside a sphere and smallest inside a sphere. One also notices that for a cylinder the $\phi$ dependence is actually restricted to a rather small range of values for $\cos \theta$ while for most polar angles $\theta$ either none or all azimuthal angles are allowed. We emphasize again that the forbidden regions are also strictly forbidden for interacting rods whose profile is no longer constant within the allowed region. 


\section{REFERENCES}

[1] L. Onsager, Ann. N. Y. Acad. Sci. 51, 627 (1949).

[2] G. Vroege and H. Lekkerkerker, Rep. Prog. Phys. 55, 1241 (1992).

[3] B. Jérôme, Rep. Prog. Phys. 54, 391 (1991).

[4] A. Poniewierski and R. Hołyst, Phys. Rev. A 38, 3721 (1988).

[5] R. Hołyst and A. Poniewierski, Mol. Phys. 65, 1081 (1988).

[6] R. Hołyst, Mol. Phys. 68, 391 (1989).

[7] B. Moore and W. McMullen, Phys. Rev. A 42, 6042 (1990).

[8] A. Poniewierski, Phys. Rev. E 47, 3396 (1993).

[9] Y. Mao, M. Cates, and H. Lekkerkerker, J. Chem. Phys. 106, 3721 (1997).

[10] Y. Mao, M. Cates, and H. Lekkerkerker, Phys. Rev. Lett. 75, 4548 (1995).

[11] Y. Mao, P. Bladon, H. Lekkerkerker, and M. Cates, Mol. Phys. 92, 151 (1997).

[12] L. Auvray, J. Physique 42, 79 (1981).

[13] K. Yaman, P. Pincus, and C. Marques, Phys. Rev. Lett. 78, 4514 (1997).

[14] K. Yaman, M. Jeng, P. Pincus, C. Jeppesen, and C. Marques, Physica A 247, 159 (1997).

[15] E. Eisenriegler, A. Hanke, and S. Dietrich, Phys. Rev. E 54, 1134 (1996).

[16] C. Gray and K. Gubbins, Theory of Molecular Fluids (Clarendon, Oxford, 1984).

[17] L. Łajtar, A. Patrykiejew, J. Penar, and S. Sokołowski, Chem. Phys. Lett. 139, 277 (1987).

[18] B. Götzelmann, A. Haase, and S. Dietrich, Phys. Rev. E 53, 3456 (1996).

[19] W. Helfrich, Z. Naturforsch. Teil C 28, 693 (1973). 


\section{FIGURES}

FIG. 1. The system under consideration consists of a fluid of monodisperse hard spherocylinders of diameter $D$ and length $L$ in contact with a spherical or cylindrical hard wall of radius $R$. We assume that the orientational distribution only depends on the normal distance $z$ and, for a spherical wall, the angle $\theta$ of the particle axis with respect to the surface normal. During the evaluation of the excess free energy the particle orientations are described in three different reference frames: the frame $x^{\prime} y^{\prime} z^{\prime}$ fixed in space, the frame $x y z$ fixed by the local normal direction, and the interparticle frame $\hat{x} \hat{y} \hat{z}$. The rotation between the latter two is described by the Euler angles $\psi_{i}, \eta_{i}$, and $\chi_{i}$ (see Eq. (8)). The different sizes of the spherocylinders indicate that the particles typically do not lie in the $x^{\prime} y^{\prime}$ plane and thus only their projection onto that plane is shown. For reasons of clarity only the polar angles $\theta^{\prime}, \theta$, and $\hat{\theta}$ are shown, but not the corresponding azimuthal angles $\phi^{\prime}, \phi$, and $\hat{\phi}$.

FIG. 2. Full density profile $\hat{\rho}^{*}(z, \cos \theta)=\hat{\rho}(z, \cos \theta) D L^{2}$ outside a sphere of radius $R / L=3$ for the bulk density $\rho_{b}^{*}=\rho_{b} D L^{2}=2$. At small distances $z$ from the wall large values of $\cos \theta$ are forbidden due to overlap. Therefore the profile is exactly zero behind this "step". In the allowed region the most prominent feature induced by the interaction between the rods is the strong increase of the density at small distances $z$. For fixed $z$ orientations close to the step, i.e., those with one end of the rod touching the wall, are favored. If the interparticle interactions were neglected the profile would be constant in the whole accessible region.

FIG. 3. Normalized orientationally averaged density $n(z)$ for fluids in contact with planar, cylindrical, and spherical walls of positive and negative curvature for a fixed bulk density $\rho_{b}^{*}=2$. The inset shows the behavior in the vicinity of the cusp which occurs at $z=L / 2$ and is followed by a rapid decay towards the bulk limit $n(z)=1$. All curves have about the same value at $z / L \simeq 0.27$ but they do not intersect exactly at one point. For better visibility only two curves are shown in the main part of the figure. The remaining profiles lie in between these two. Close to the wall the number density is larger for positive curvature. Inside a sphere $n(z)=0$ for very small $z$ because the centers of the rods cannot come arbitrarily close to the wall. Small kinks in the small $z$ range that are caused by the numerical discretization were removed by fitting a smooth curve to the raw data.

FIG. 4. Uniaxial nematic order parameter $Q_{20}$ as defined in Eq. (38) for a fluid outside a sphere of radius $R / L=5$ for different densities. The tendency for parallel orientations, as indicated by negative values of $Q_{20}$, becomes more pronounced and longer ranged with increasing density. $Q_{20}(z=0)=-0.3154$.

FIG. 5. Order parameter $Q_{20}$ at fixed density $\rho_{b}^{*}=2$ for different wall geometries. Parallel orientations, i.e., negative values of $Q_{20}$ are more favored by negative curvatures.

FIG. 6. Biaxial order parameter $Q_{22}$ (see Eq. (38)) for cylindrical walls of positive and negative curvature at different bulk densities. The most probable orientations for positive and negative values of $Q_{22}$ are indicated in the sketches. The strong increase of the decay length with density for $R / L=-5$ signals the formation of a nematic wetting layer. 
FIG. 7. Density dependence of the surface tension for different wall geometries and curvatures. In the ideal limit $\beta \gamma / \rho_{b} L$ takes on the density independent values 0.25 for $1 / R \geq 0$ (cylinders, spheres, and planar wall), and $0.2492(0.2497)$ for a sphere (cylinder) with $R / L=-5$. The differences between these three values are not visible on the scale of the figure.

FIG. 8. Curvature dependence of the surface tension for (a) spheres and (b) cylinders at various densities. In the limit of non-interacting rods $\left(\rho_{b}=0\right)$ there is a slight decrease of $\gamma$ for negative curvature, which is hardly visible on this scale. Taking into account the interparticle interactions leads to a substantial enhancement of the curvature dependence already for $\rho_{b}^{*}=1$. We have interpolated smoothly between the 7 data points calculated for each curve.

FIG. 9. Geometry of two overlapping rods in the limit $D / L \rightarrow 0$. Only one half of each rod is shown. Their centers lie on the $\hat{z}$ axis and have a distance $r$. In order to overlap both rods must lie approximately in the same plane (a). Part (b) shows a vertical projection from which the allowed azimuthal range $\Delta \hat{\phi}_{12}=2 D / p$ can be determined. Rod 2 is drawn in the two positions for which it just touches rod 1.

FIG. 10. Illustration of the cases that must be distinguished for the determination of the minimum $\left(x_{\min }=\cos \hat{\theta}_{2}^{\max }\right)$ and maximum $\left(x_{\max }=\cos \hat{\theta}_{2}^{\min }\right)$ value of $x_{2}$ so that two rods overlap for given $x_{1}=\cos \theta_{1}$ and $r$. The appropriate expressions for $x_{\min }$ and $x_{\max }$ for the regions $\mathrm{A}, \mathrm{B}$, $\mathrm{C}$, and $\mathrm{D}$ are given in the main text. In the unlabeled region containing the inset overlap is not possible. The inset shows an example from region $\mathrm{B}$ where the end of rod 2 touches rod 1 at the minimum angle and vice versa at the maximum angle.

FIG. 11. Geometries for the determination of the allowed orientations at curved walls. (a) Outside a spherical wall the rod touches the wall with its end if $r>r_{c}(\operatorname{rod} 1)$, but tangentially if $r<r_{c}$ (rod 2). Inside a sphere only end contact can occur (rod 3). From this figure Eqs. (B1) and (B2) can be derived. (b) Projection of the corresponding problem for a cylindrical wall onto the plane perpendicular to the cylinder axis. Equations (B3) and (B34) can be derived using $x_{P}=\frac{L}{2} \sin \theta \cos \phi_{c}$ and $z_{P}=\frac{L}{2} \cos \theta$.

FIG. 12. In calculating the accessible orientational space for a rod outside a cylinder different expressions, presented in the main text, apply in the regions labeled A, B, C, and D for the maximum azimuthal angle $\phi_{c}$ at given values of $x=\cos \theta$ and $r$. The figure corresponds to the case $R / L=1.3$, but its topology is the same for all radii. Only for configurations corresponding to region $\mathrm{D}$ the rod touches the cylinder tangentially. For larger values of $R$ as actually used in the calculations presented above $r_{c}$ is closer to $R$ and the regions $\mathrm{C}$ and $\mathrm{D}$ are much smaller.

FIG. 13. Accessible orientational space for a rod near walls of different geometries at a fixed distance $z / L=0.2$ and radius $|R| / L=3$. Spatial angles to the left of the lines are allowed, those to the right are forbidden by the presence of the wall. There is no dependence on the azimuthal angle $\phi$ for spherical walls. For a cylinder at $\phi=0(\phi=\pi / 2)$ the wall is effectively spherical (planar) which explains the common end points of the various lines. 


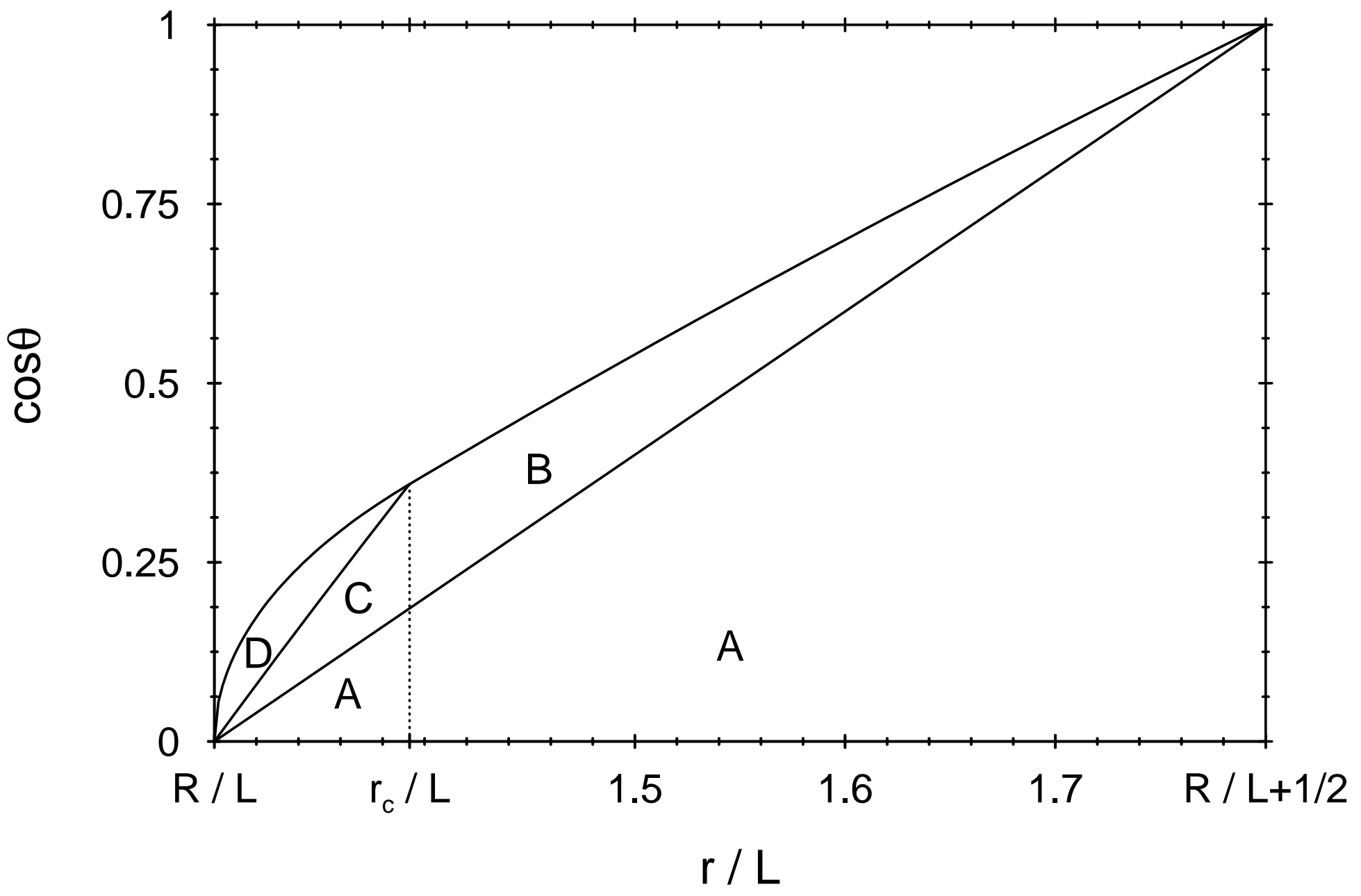

Fig. 12 


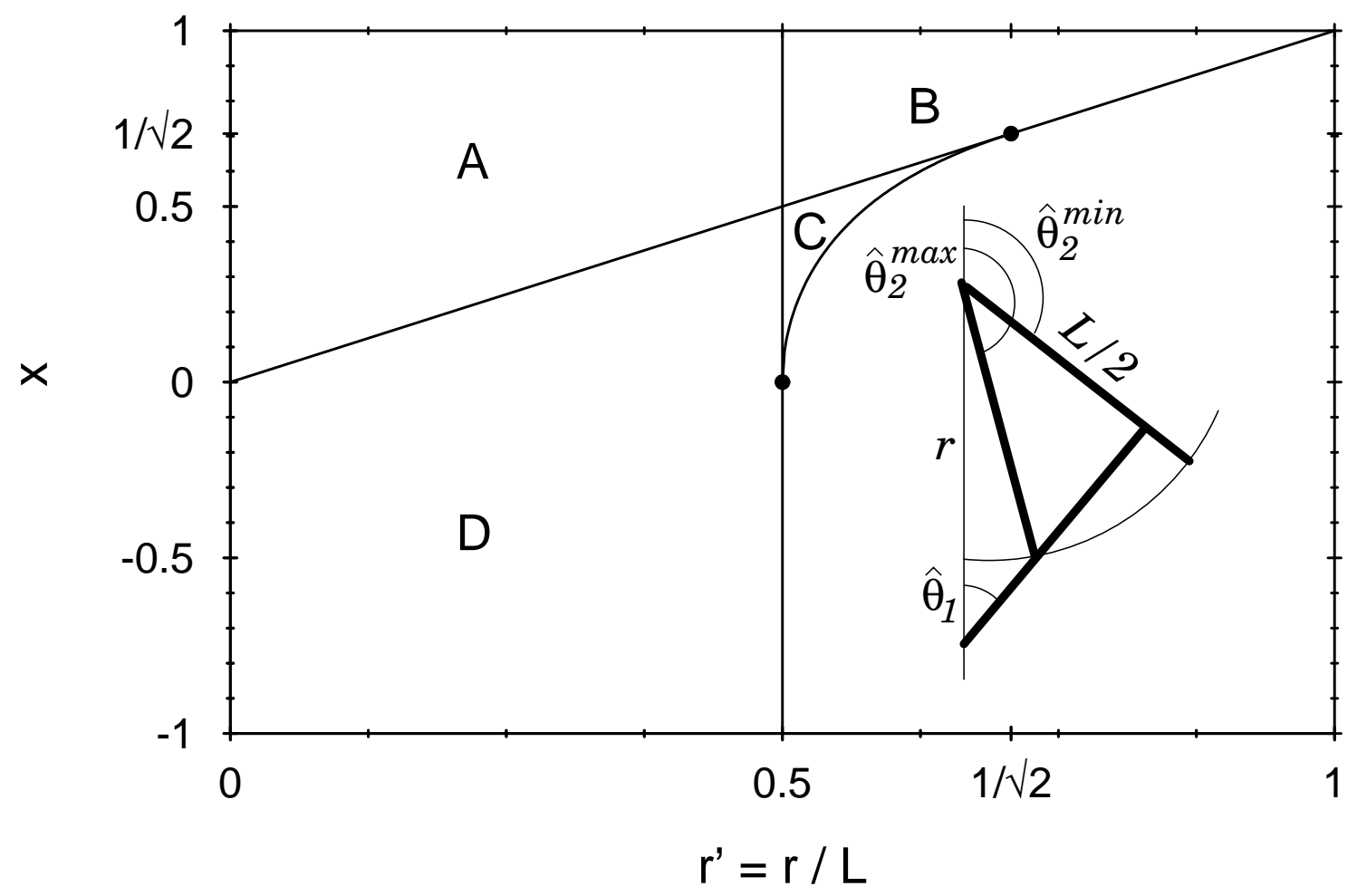

Fig. 10 

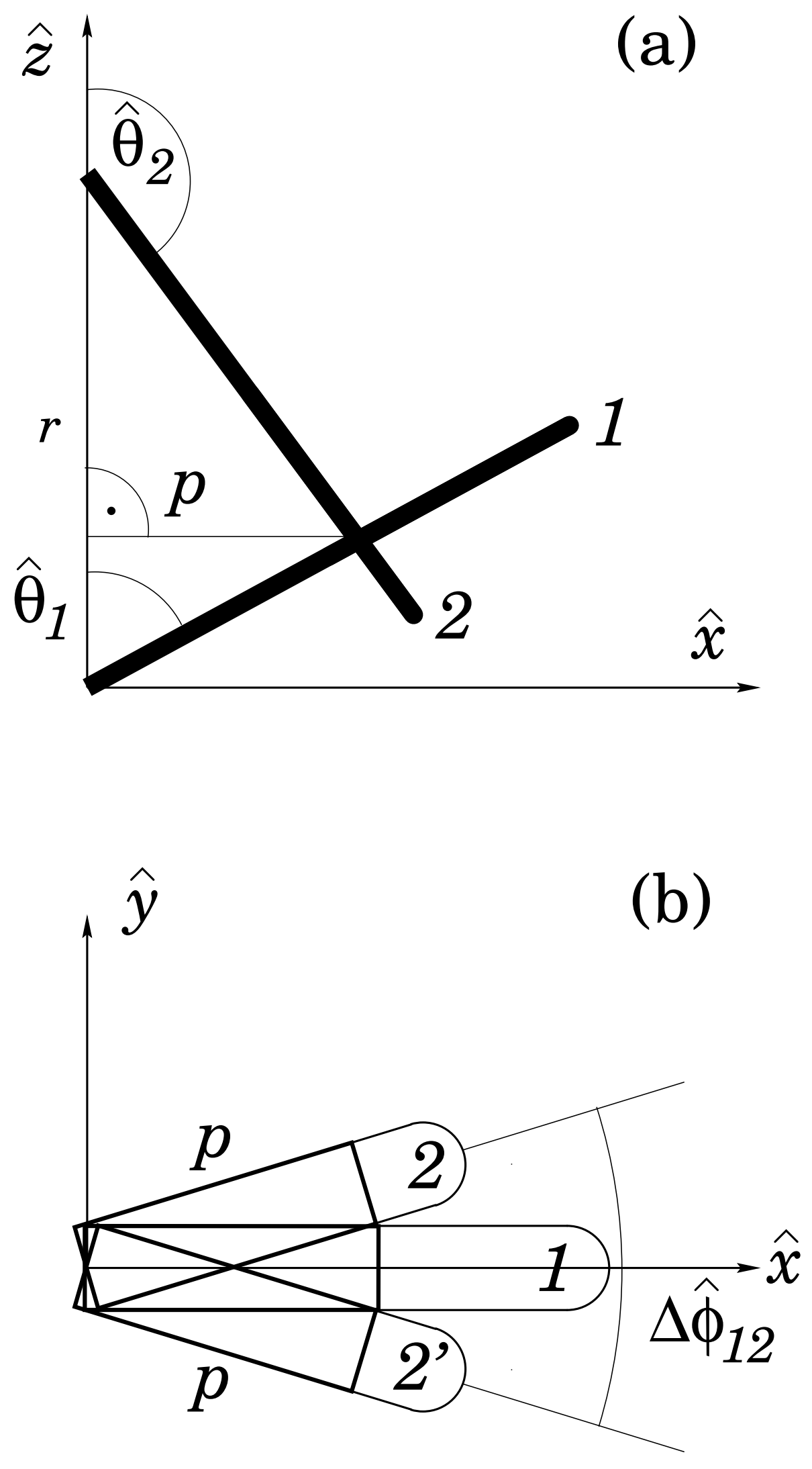

Fig. 9 


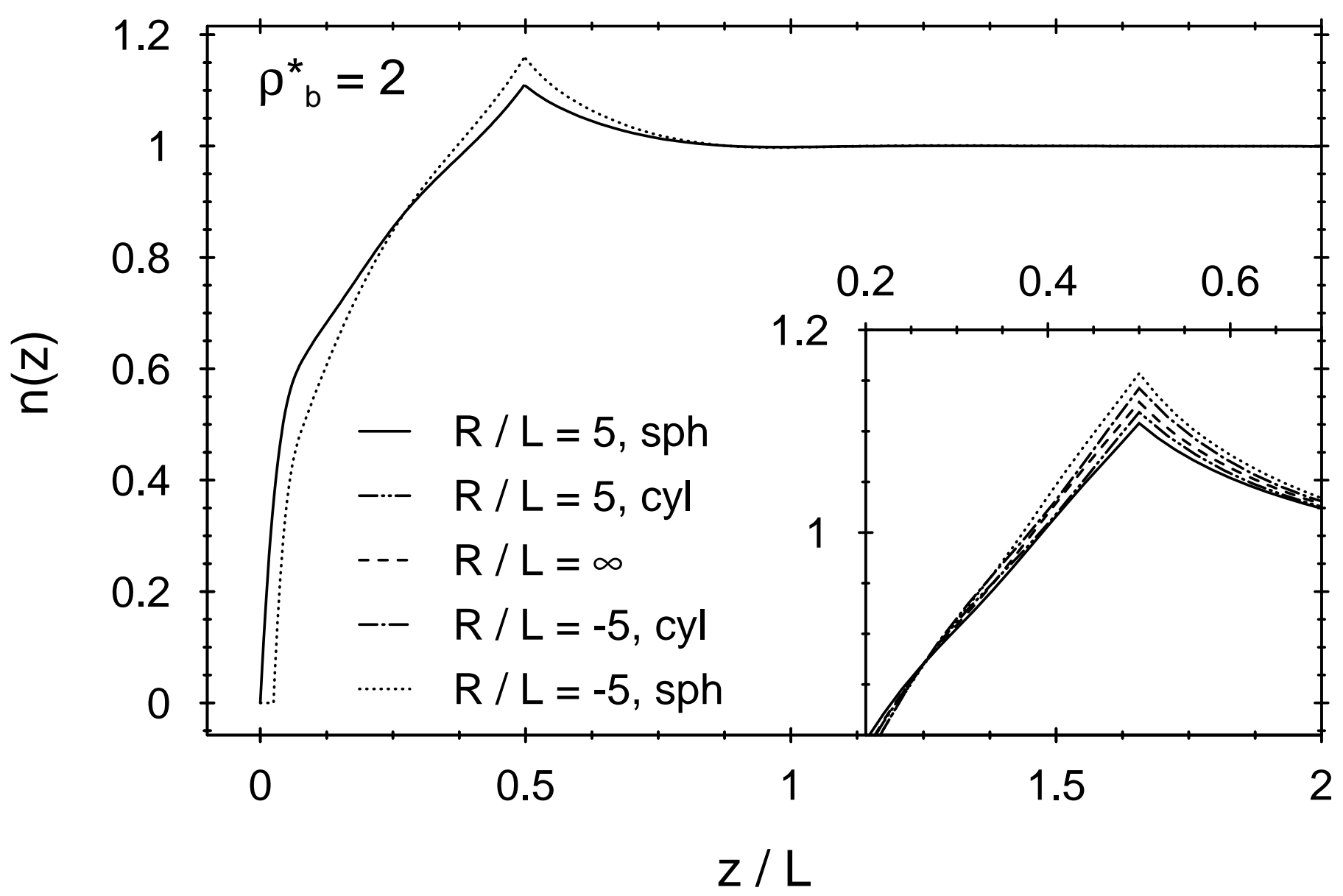

Fig. 3 


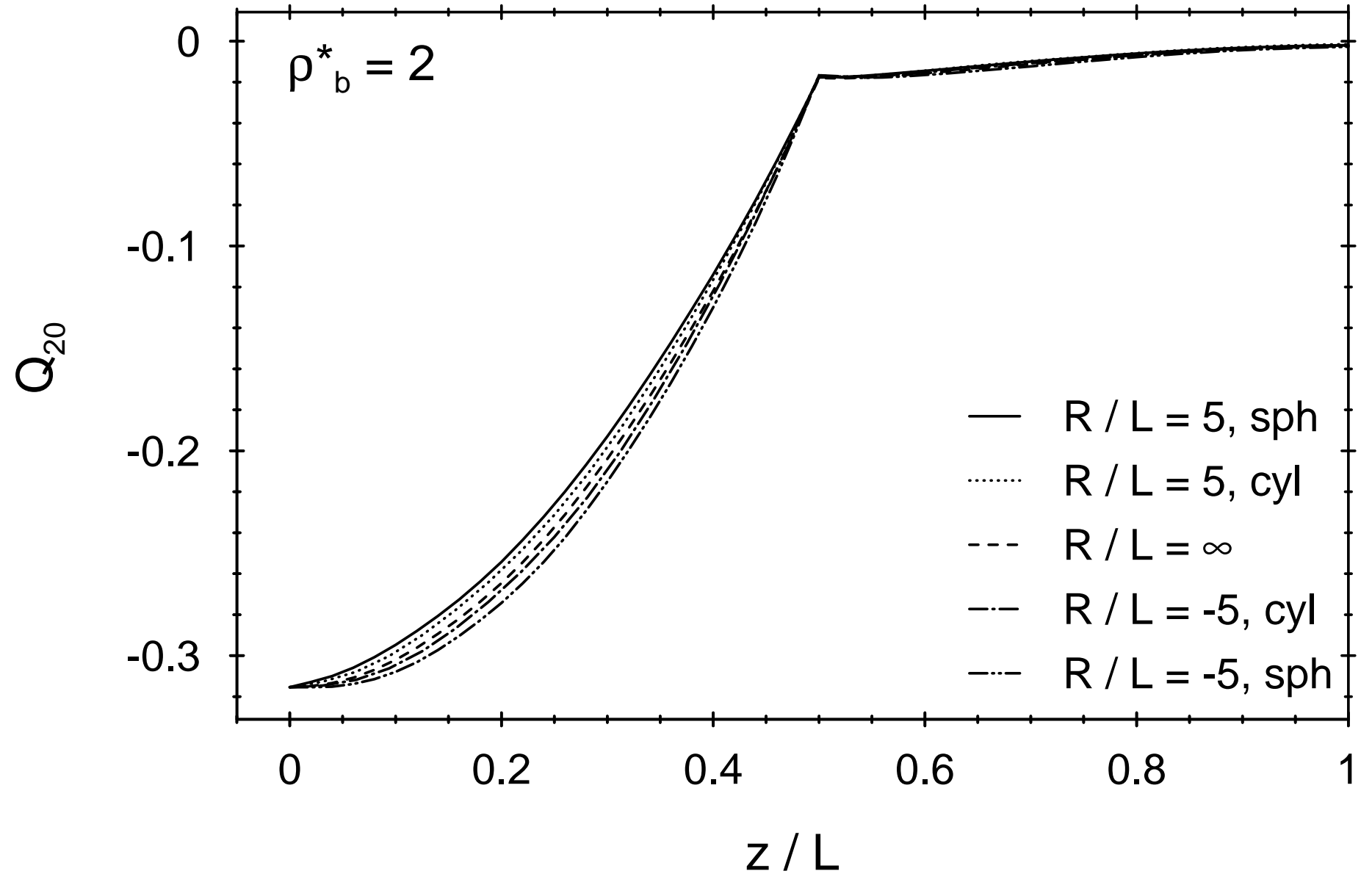

Fig. 4 


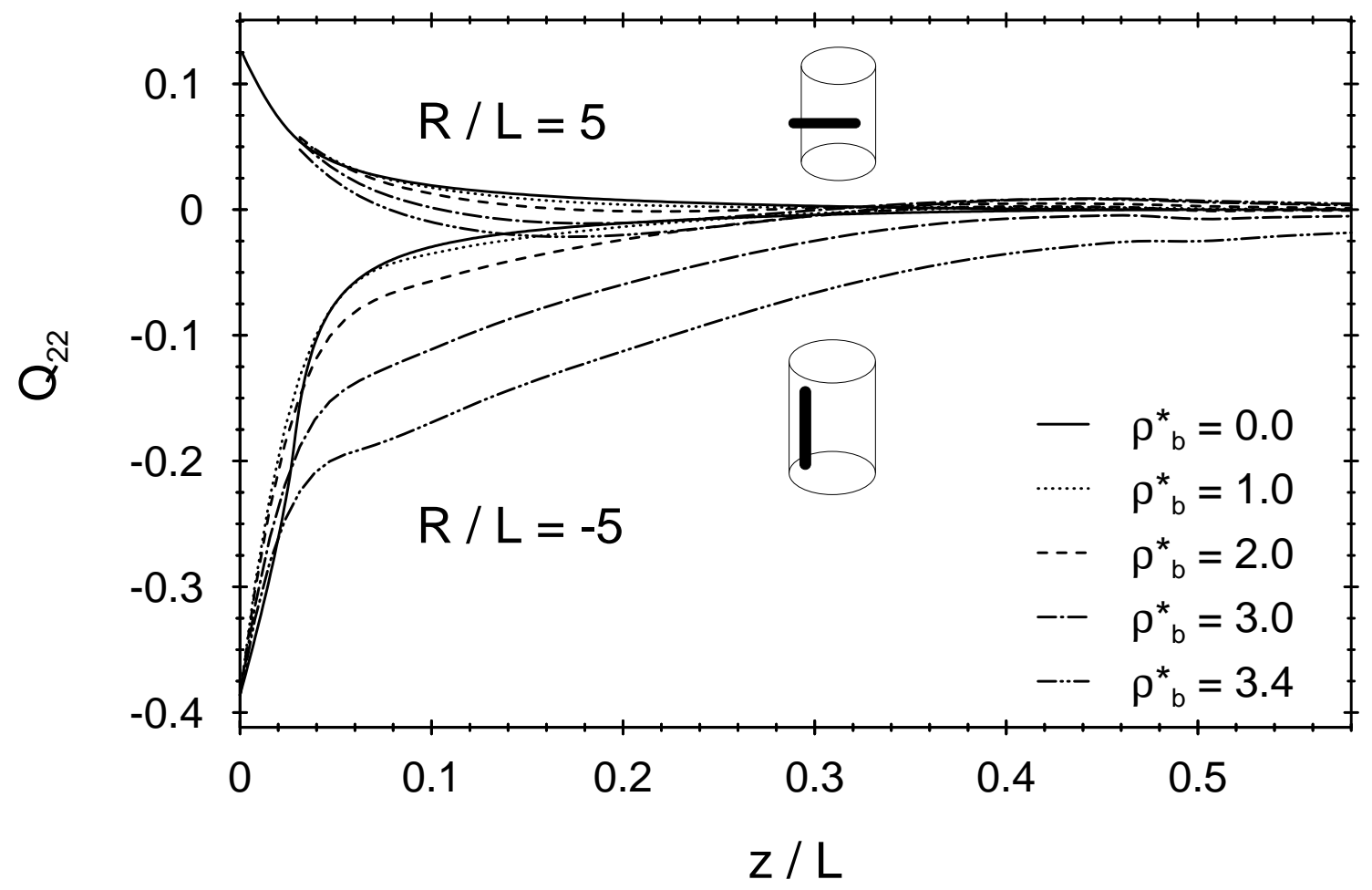

Fig. 6 


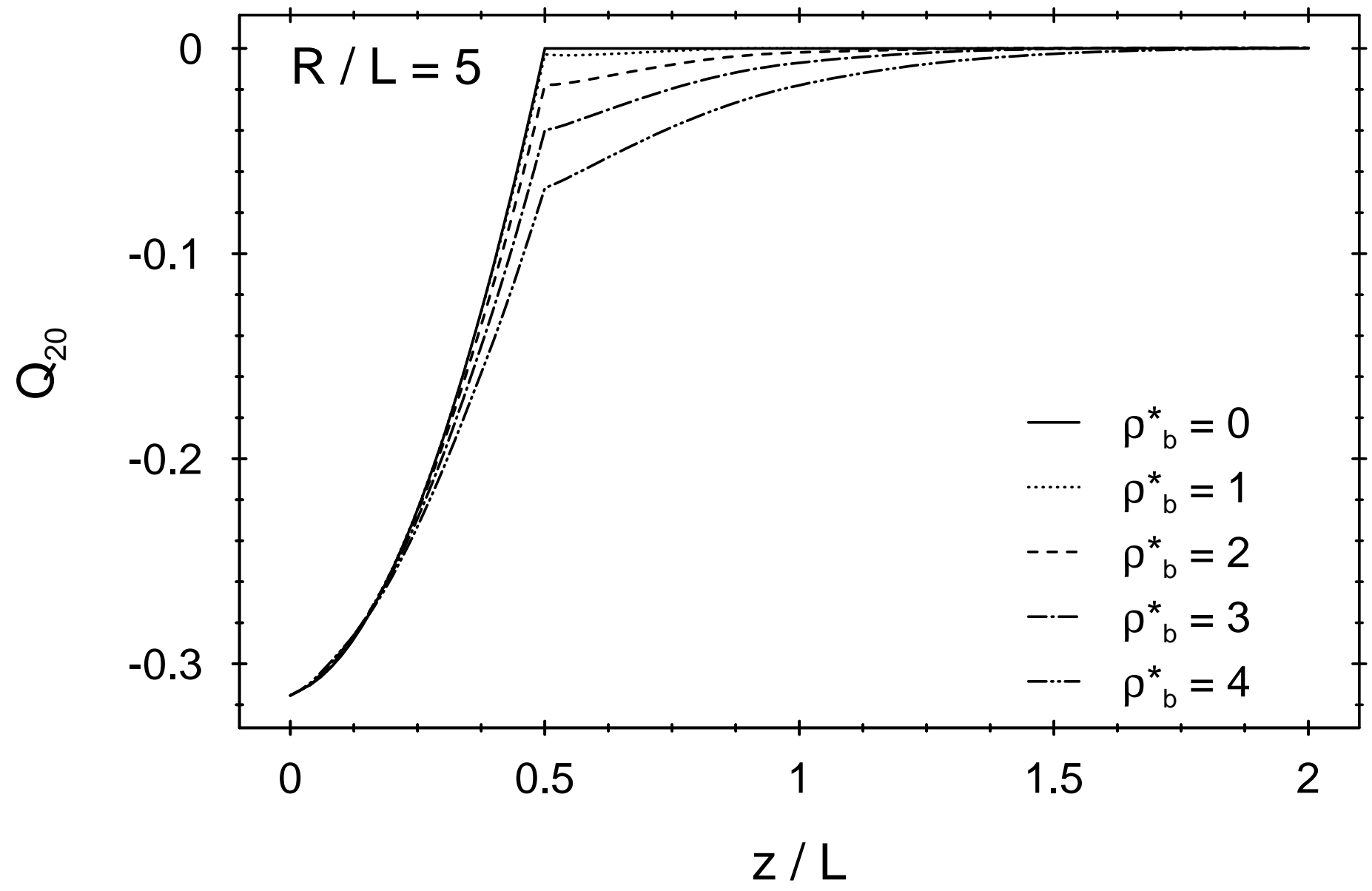

Fig. 5 


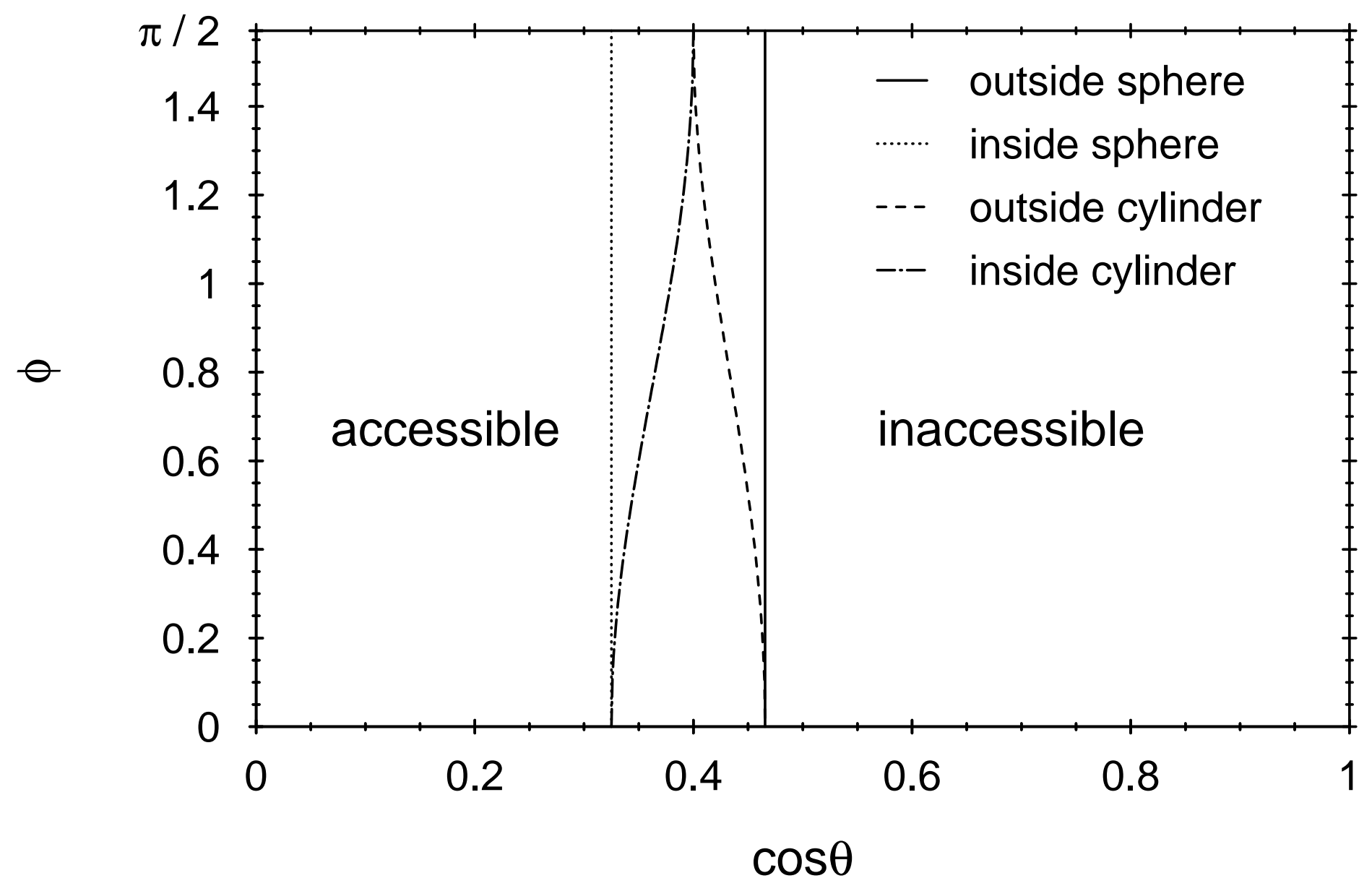

Fig. 13 


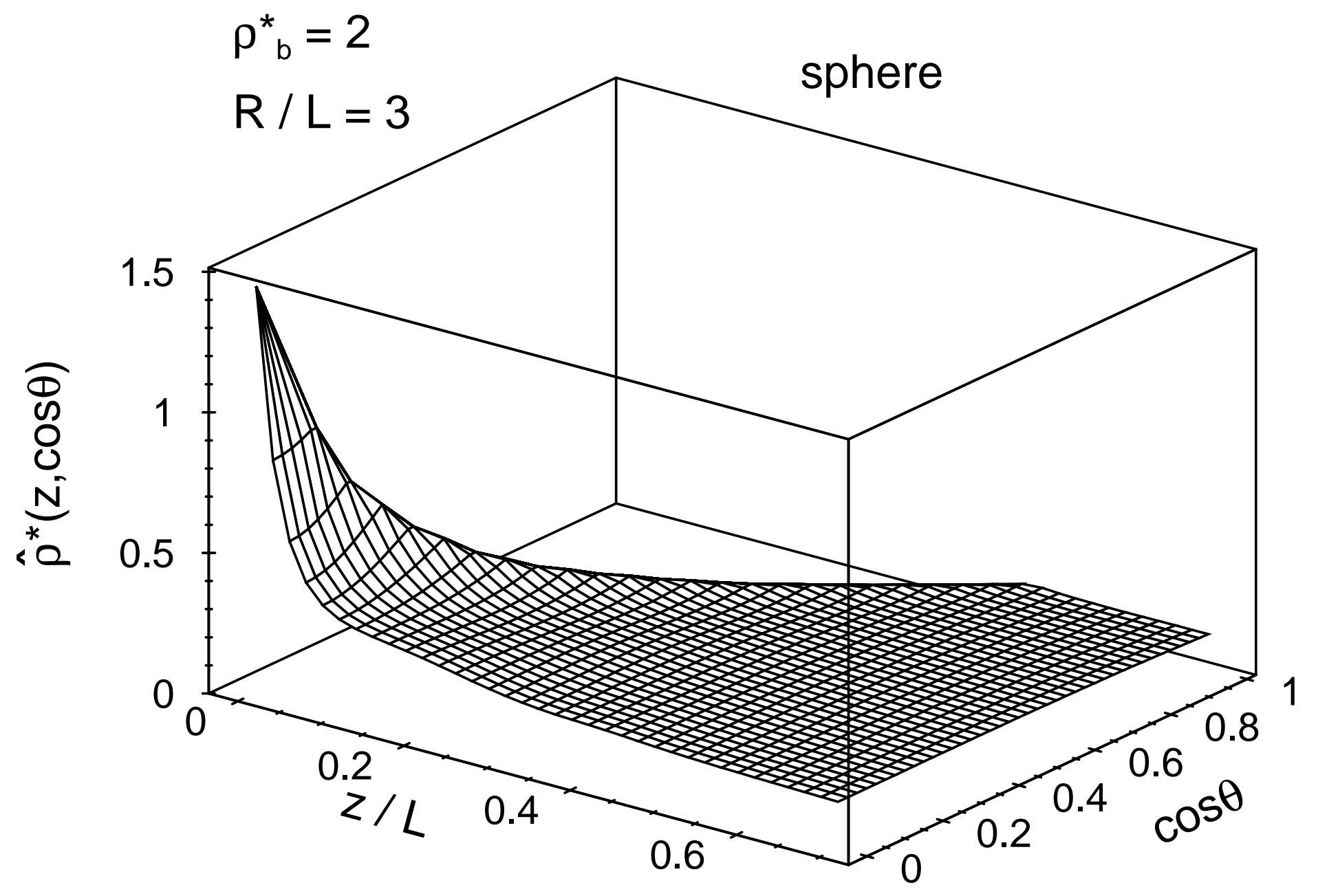

Fig. 2 


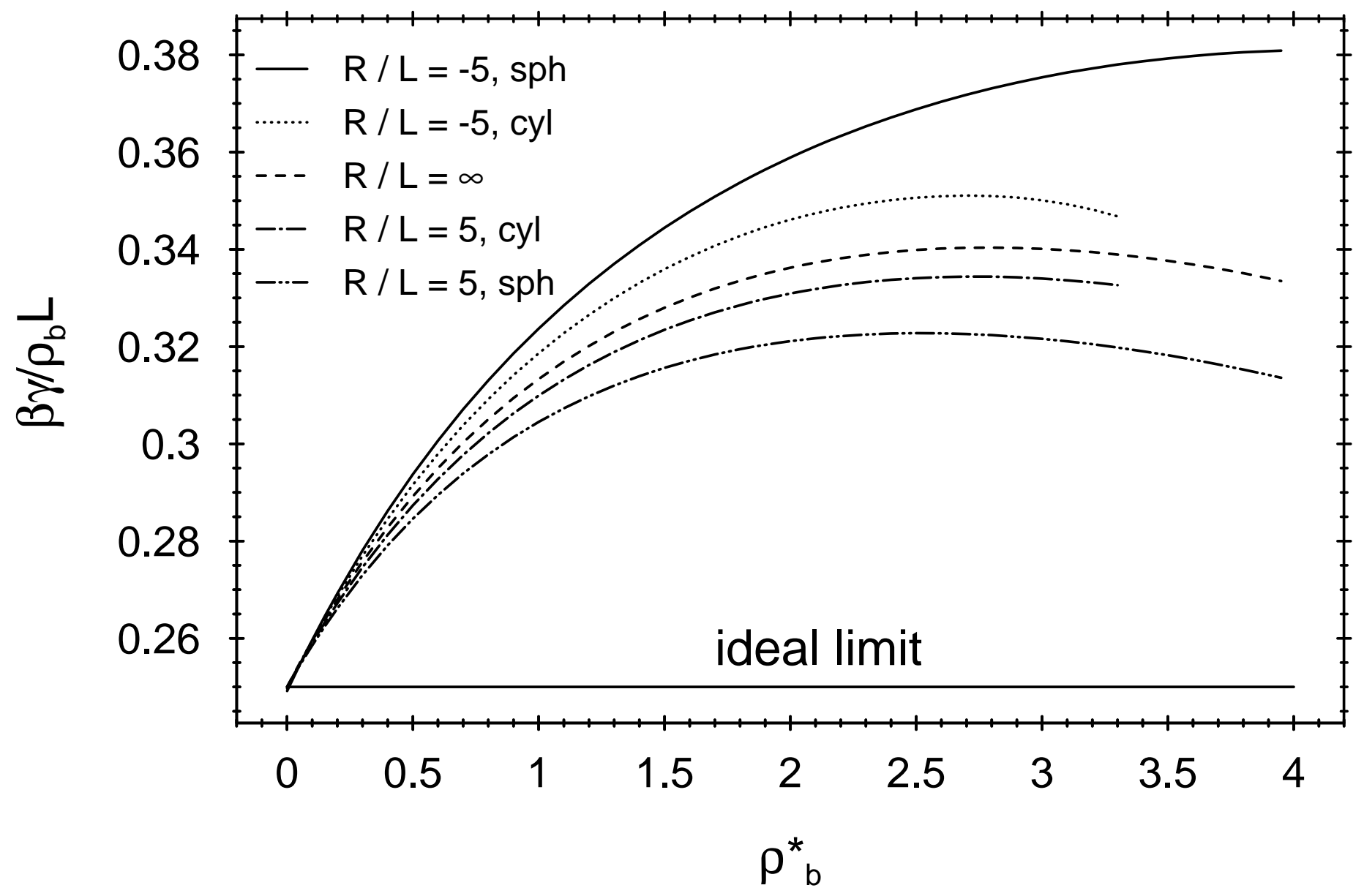

Fig. 7 


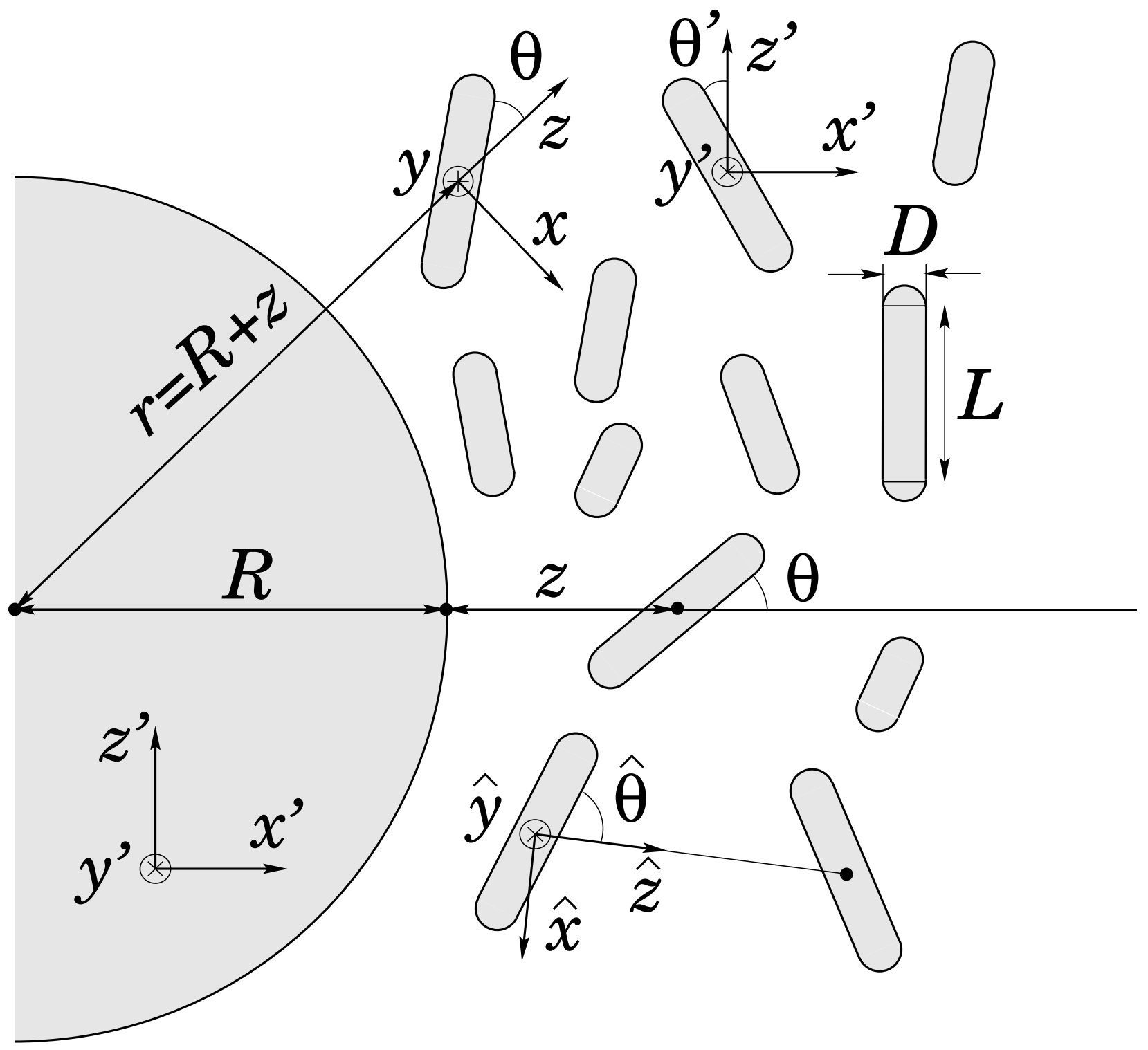

Fig. 1 


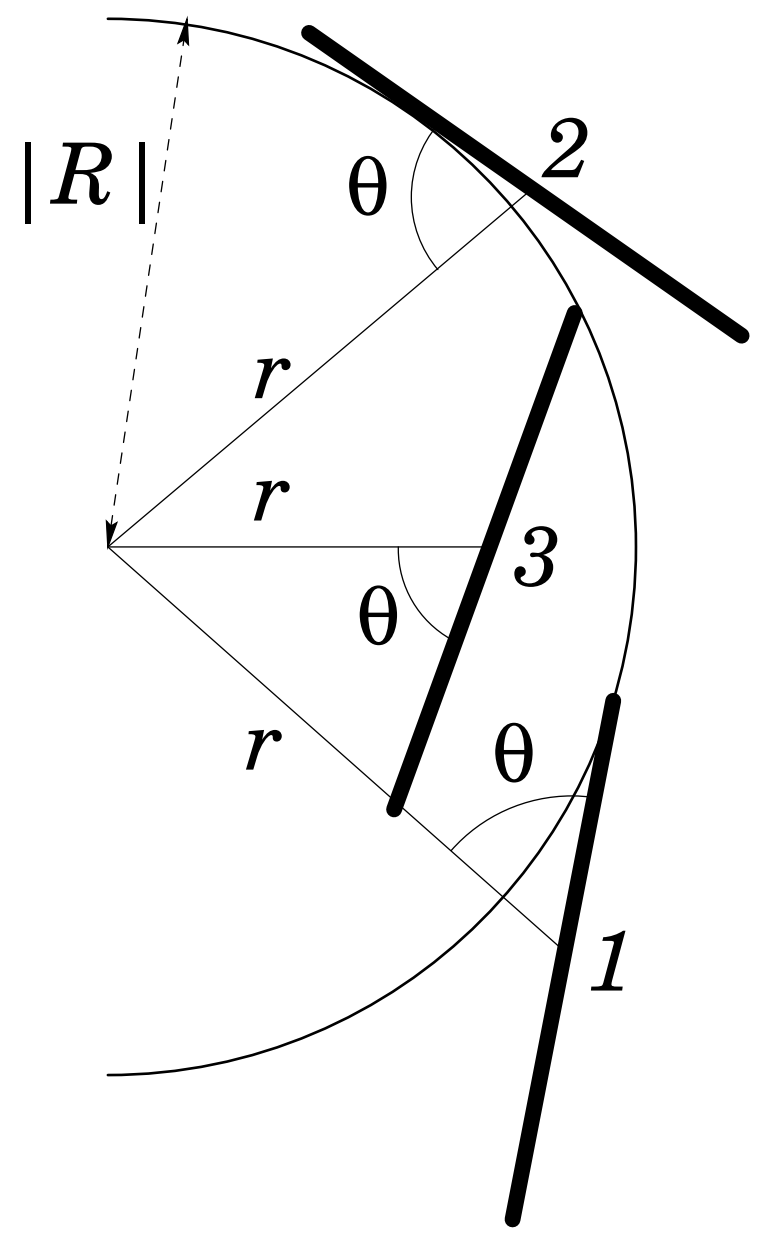

(b)

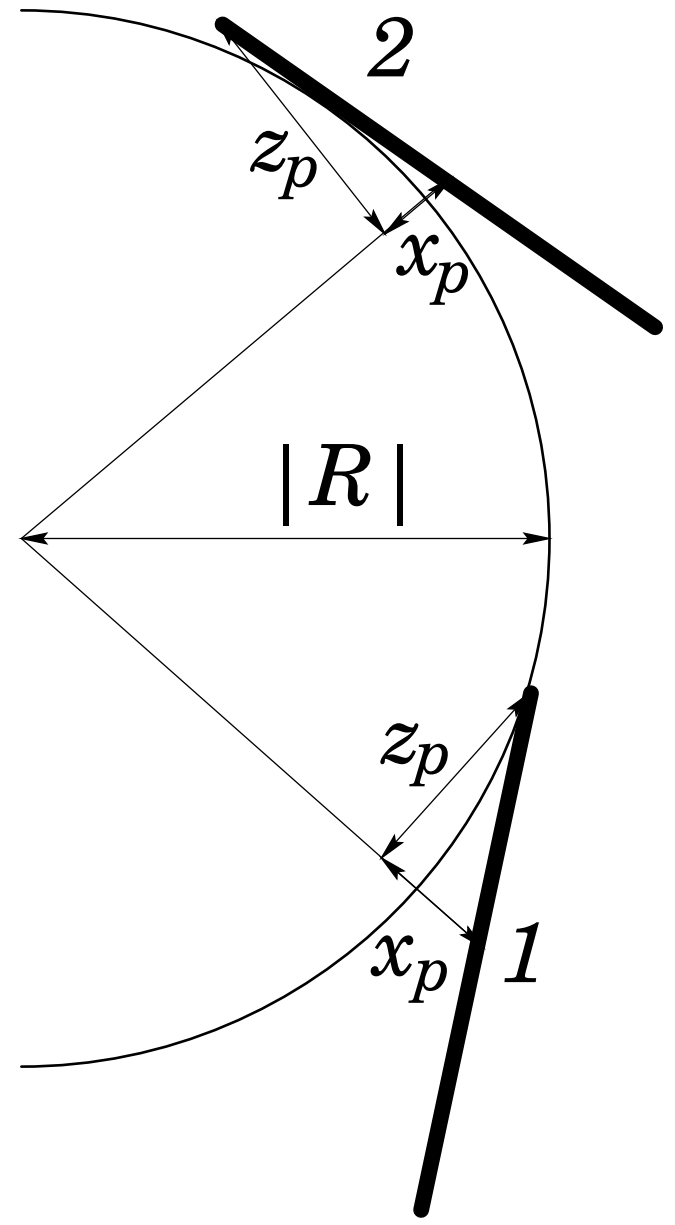

Fig. 11 